\title{
Energy absorption characteristics of three-layered sandwich panels with graded re-entrant hierarchical honeycombs cores
}

\author{
H.L. Tan ${ }^{\text {a }}$, Z.C. He ${ }^{\mathrm{a}^{*}}$, E. $\mathrm{Li}^{\mathrm{b}}$, X.W. Tan ${ }^{\mathrm{c}}$, A.G. Cheng ${ }^{\mathrm{a}}$, Q.Q. Li ${ }^{\mathrm{d}}$ \\ ${ }^{a}$ State Key Laboratory of Advanced Design and Manufacturing for Vehicle Body, Hunan University, \\ Changsha, 410082 P. R. China \\ ${ }^{\mathrm{b}}$ School of Science, Engineering \& Design, Teesside University, Middleborough, United Kingdom \\ ${ }^{c}$ Guangxi University of Science and Technology, Liuzhou 545006, PR China \\ ${ }^{\mathrm{d}}$ College of Automotive and Mechanical Engineering, Changsha University of Science and Technology, \\ Changsha, 410114 P. R. China
}

\begin{abstract}
Two innovative re-entrant hierarchical sandwich panels constructed by substituting the cell walls of re-entrant honeycombs with isotropic regular hexagon substructure (RHH) and equilateral triangle substructure (RHT) are proposed in this paper, and their crashworthiness performance has been investigated systematically. Based on the Euler beam theory, the Young's moduli of units of re-entrant hierarchical sandwich panels are derived. A comparison between the re-entrant hierarchical sandwich panels and re-entrant sandwich panel has been conducted to study their energy absorption ability. Furthermore, the parametric analysis based on numerical analysis has been carried out to discuss the effects of the gradient, arranged orientation of hierarchical sandwich cores and the impact velocities. Results show that both the two proposed sandwich panels can greatly improve the in-plane stiffness and the energy absorption capacity, and the energy absorption mechanisms are discussed. In addition, the graded sandwich panels can effectively reduce initial peak force under quasi-static compression, and the configuration of large- medium -small (LMS) performs the best when under high impact velocity. The cross-arranged cores can significantly improve the impact resistance ability of RHT sandwich panels. This work provides a new solution for designing lightweight sandwich structures.
\end{abstract}

Key words: sandwich panel; graded honeycombs; crashworthiness analysis; energy absorption; hierarchical honeycomb.

\footnotetext{
* Corresponding author. Tel./fax: +86 18817119011.

E-mail address: hezhicheng815@163.com
} 


\section{Introduction}

Sandwich structures are generally composed of two high strength plates and a relatively thick and low-density core, which have typical characteristics of light weight, high rigidity and high strength. Because of its excellent mechanical performance, sandwich structures are widely used in aerospace, automobile and marine industries.

In recent years, there has been an increasing amount of literature on the energy absorption ability and structural crashworthiness of sandwich structures. Yang et al. [1] proposed a novel lightweight double-sine corrugated sandwich structure, and conducted the quasi-static compression. They concluded that the double-sine corrugated core sandwich panels can significantly improve the structural crashworthiness compared with the regular triangular and sinusoidal corrugated core sandwich panels. Hou et al. [2] investigated the relationship between the sandwich parameters and the crashworthiness performance of trapezoidal and triangular core sandwich panels under low-velocity impact. Reddy et al. [3] studied the response and energy absorption capacity of cellular panels which consist of silk-cotton wood skins and aluminum honeycomb core. Zhang et al. [4] analyzed the indentation response and energy absorption property of the honeycomb sandwich panels subjected to drop-weight impact by numerical simulation and experiment. Qin et al. [5] proposed an analytical model to predict the dynamic response of square sandwich plate struck by a heavy mass with low-velocity. Liu et al. [6] studied the energy absorption behaviors of composite sandwich structures which are composed of a coconut 
mesocarp core and two glass fiber reinforced plastics sheets. Hu et al. [7] designed a novel lattice truss sandwich panel which is made up of orthogonal corrugated lattice trusses, and carried out a series of experiments to reveal the failure modes. Li et al. [8] explored the strength and failure mode of carbon fiber reinforced corrugated-core sandwich cylinders. Nia et al. [9] investigated the ballistic limit velocity of sandwich structures with wired core and face-sheets numerically and experimentally. Mahmoudabadi et al. [10] studied the energy absorption abilities of sandwich panels with metal honeycomb cores under quasi-static punch loading, and found that the effect of core thickness on energy absorption capacities is greater than that of facesheet thickness.

In addition, the introduction of structural hierarchy has a great effect on the crashworthiness. Wen et al. [11] constructed a novel hierarchical honeycomb inspired by pomelo peel and studied the energy absorption capabilities of this structure. Marian et al. [12] investigated the behavior of a hierarchical sandwich structure consisted of self-reinforced poly-ethylene terephthalate (PET) combined with PET foam and performed the specific energy absorption in quasi-static loading conditions. An et al. [13] revealed compression behaviors of luffa-sponge-like hierarchical structures, and found that the specific energy absorption of hierarchical structures is much greater than pure aluminum cylinder structures. Tsang et al. [14] proposed the hierarchical tubular section inspired by biological materials and revealed the introduction of hierarchy can improve the impact energy absorption capability. Sun et al. [15] designed the hierarchical lattice tubes with lattice sandwich walls and carried 
out the in-plane compression experiments to reveal the collapse modes and folding mechanisms. Zhang et al. [16] proposed a novel hierarchical circular tube to enhance the crashworthiness performance, and found that the proposed hierarchical structure exhibits obvious advantages for energy absorption efficiency. Li et al. [17] studied the crushing mechanisms of the tubes with hierarchical section, and concluded that the hierarchical tube is a more weight-efficient energy absorber. Tan et al. [18] studied the crashworthiness of two novel hierarchical honeycombs, and derived the plateau stress based on a two-scale method.

The auxetic structures have also attracted a lot of attention because of their excellent physical properties and extensive application. Jin et al.[19] proposed auxetic re-entrant cell honeycomb cores and investigated the dynamic responses and blast resistance of the sandwich structures, and found that the new sandwich panel have a higher ability of resisting deformation. Lu et al.[20] calculated the in-plane Poisson's ratio and Young's modulus of the novel auxetic structure by adding a narrow rib in traditional re-entrant honeycomb and found that the novel structure exhibit enhanced Young's modulus through the analytical and FE analysis. Qi et al. [21] mitigated the damage of shock loads form contact detonations of high explosives by a new protective system employing the auxetic honeycomb-cored sandwich structures. Liu et al. [22] studied the impact response of the auxetic honeycomb and described the characteristics of crushing patterns and modes of the honeycomb, and compared the energy absorption performances of the auxetic honeycomb and the hexagonal honeycomb. Fu et al. [23] concluded that both the in-plane Young's modulus and 
buckling strength of the novel auxetic honeycomb are higher than the normal reentrant hexagonal honeycomb by using the theoretical analysis and numerical simulations. Duc et al. [24] investigated the vibration and dynamic response of sandwich auxetic cylindrical panels using analytical solution. Lan et al. [25] compared several sandwich panels with different cores to study the dynamic response, and discovered that the cylindrical panels with designed auxetic honeycomb cores have a better performance than other cores in resisting blast loadings. Guo et al. [26] explored the energy absorption and deformation behaviors of re-entrant auxetic lattice cylindrical shells. Guo et al. [27] analyzed the dynamic properties of the double arrowed auxetic honeycomb, and found that the crushing strength rises with the increase of impact velocity and the relative density.

Functionally graded materials characterized by a gradual change in a prescribed volume have been frequently used as the core material of sandwich structures. Li et al. [28] investigated the thermal post-buckling behavior of sandwich structures with functionally graded honeycomb cores. Taylor et al. [29, 30] combined both hierarchy and functional grading and developed a set of analytical models to describe these honeycombs based on beam mechanics and the transform section method. Pradhan et al. [31] proposed the thermo-mechanical vibration analysis of functionally graded beams investigated the parametric study on the vibration response of these structures. Xiao et al. [32] studied the compression behavior of graded auxetic reentrant honeycomb, and presented the deformation mode, crushing stress and Poisson's ratio distribution. Sun et al. [33] introduced functionally graded foam fillers to thin-walled 
structures to improve the crashworthiness.

The requirement for lightweight structure with excellent energy absorption capacity is urgently needed. Sandwich panels with excellent mechanical performance play an important role in engineering application. Additionally, the structures with low initial peak load can be used in vehicle design such as bumper system and crash box to reduce the injury of the passenger in traffic accidents. As has been noted, a lot of works have been done in the introduction of hierarchy, the study of auxetic structures and the functionally graded structures to improve the energy absorption capacity. However, most of work was only focused on one of these characteristics, and few attentions are to combine them. The purpose of this paper is to propose novel sandwich panels which combine the hierarchy, characteristic of auxetic structures and gradient to enhance the mechanical properties of the sandwich panels. Section 2 gives the geometric configuration and the finite element modeling of the hierarchical sandwich panels. Section 3 derives the Young's moduli of units of re-entrant hierarchical sandwich panels based on the Euler beam theory. Section 4 conducts a comparison between the re-entrant hierarchical sandwich panels and re-entrant sandwich panel to study their energy absorption ability, and the parametric analysis based on numerical analysis has been carried out to discuss the effects of the gradient, arranged orientation of hierarchical sandwich cores and impact velocities. Several conclusions are made in Section 5. 


\section{Geometric configuration and finite element modeling}

\subsection{Geometric configuration}

A sandwich panel usually consists of two face sheets and a core. As shown in Fig. 1, the geometric and dimension of three types units of sandwich cores are described. The basic form of re-entrant honeycomb (RH) is presented in Fig. 1(a), and the re-entrant hierarchical honeycombs constructed with regular hexagon substructures (RHH) and equilateral triangle substructures (RHT) instead of cell walls are provided in Fig. 1(b) and Fig. 1(c), respectively. $l_{h}$ and $l_{t}$ are the edge length of regular hexagon substructure and equilateral triangle substructures. $t_{0}, t_{h}$ and $t_{t}$ are the thickness of RH, RHH and RHT, respectively. $1+N_{h 1}$ and $1+N_{h 2}$ mean the number of hexagon substructures along the vertical orientation and inclined direction of the unit cell of RHH. $N_{t 1}$ and $N_{t 2}$ denote the number of triangle substructures along the vertical orientation and inclined direction of the unit cell of RHT.

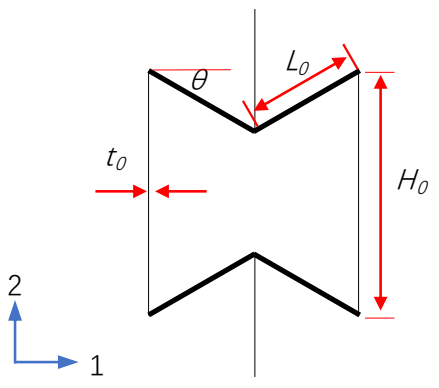

(a) RH

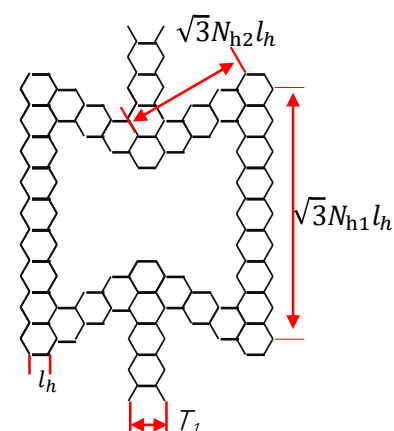

(b)RHH

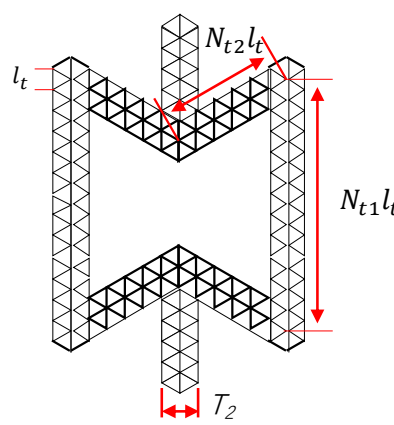

(c)RHT

Fig. 1. Diagrammatic sketches of three types units of the core of sandwich panels:

(a) RH; (b) RHH; (c) RHT.

The relative densities of three types units are given by: 


$$
\begin{aligned}
& \bar{\rho}=\frac{\left(H_{0}+2 L_{0}\right)}{\sqrt{3}\left(H_{0}-L_{0} / 2\right)} \frac{t_{0}}{L_{0}} \\
& \overline{\rho_{h}}=\frac{10\left(N_{h 1}+2 N_{h 2}-2\right)}{3 \sqrt{3} N_{h 2}\left(2 N_{h 1}-N_{h 2}\right)} \frac{t_{h}}{l_{h}} \\
& \overline{\rho_{t}}=\frac{14\left(N_{t 1}+2 N_{t 2}-\frac{20}{7}\right)}{\sqrt{3} N_{t 2}\left(2 N_{t 1}-N_{t 2}\right)} \frac{t_{t}}{l_{t}}
\end{aligned}
$$

where $\bar{\rho}, \overline{\rho_{h}}, \overline{\rho_{t}}$ are the relative densities of the RH, RHH and RHT, respectively.

Fig. 2 presents the geometric dimensions of the cores of three types sandwich panels. The core is composed of two interface sheets and three layers of honeycomb cores. The critical geometric parameters of the core include the height of honeycomb cores $H_{c}$, the thickness of face sheets $T_{1}$ and interface sheets $T_{2}$. In order to compare the mechanical performance of proposed sandwich structures, the geometric dimensions of all types of cores with same material are the same, which means the identical height and the same thickness of interface sheet.

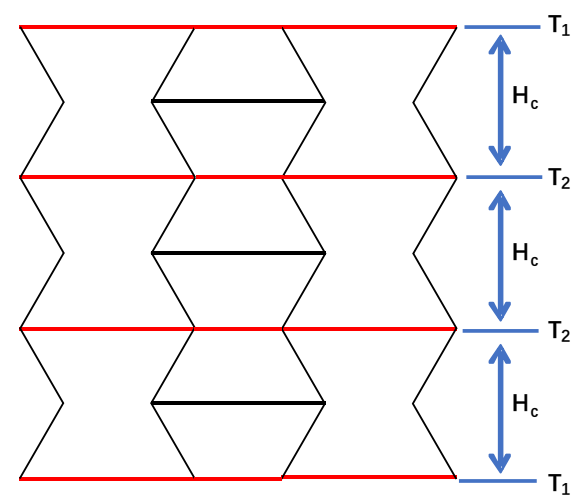

(a) RH

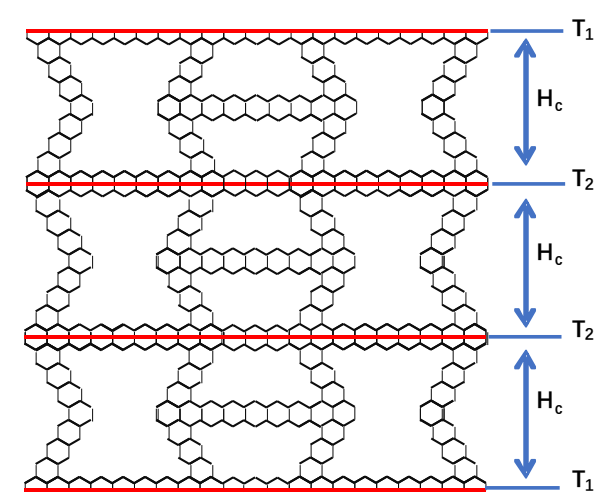

(b) RHH

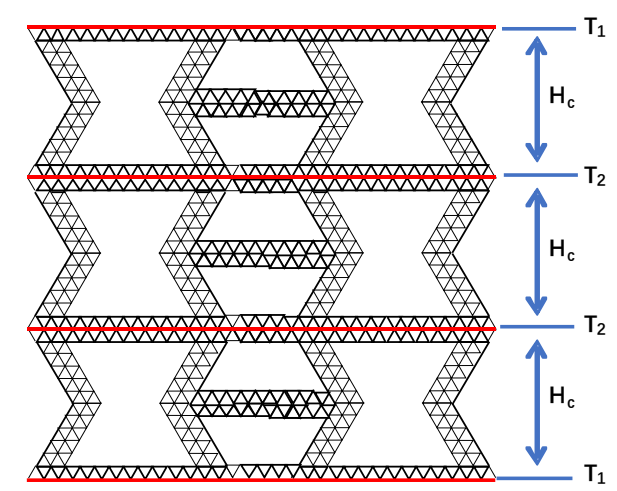

(c)RHT

Fig. 2. Geometrical configuration of three-layered sandwich panels: (a) RH; (b)RHH; (c)RHT. 


\subsection{Crashworthiness assessment}

In this study, several well-known terminologies including the energy absorption (EA), the specific energy absorption (SEA) and the mean crushing force (MCF) are used to evaluate the crashworthiness of these sandwich structures.

The energy absorption (EA) means the total strain energy absorbed by structures, and can be obtained by:

$$
E A=\int_{0}^{d} F(x) d(x)
$$

where $F(x)$ is the impact force, and variable $x$ denotes the crushing distance. Apparently, higher EA means better energy absorption of a structure.

The SEA is the absorption of energy of per unit mass which is used to evaluate the energy-absorbing efficiency. The SEA is defined as:

$$
S E A=\frac{E A}{m}
$$

where $m$ is the mass of the sandwich structure.

The mean crushing force $(\mathrm{MCF})$ is an important design variable for a structure, a higher MCF can absorb more energy, and can be derived from:

$$
M C F=\frac{E A}{d}
$$

\subsection{Finite element modeling}

The numerical models of these sandwich structures are established by using the explicit nonlinear finite element software Ls-dyna, and the mechanical performance of these novel structures are systematically investigated. In the simulations, two different arranged orientations of three sandwich panels are presented: regularly-arranged and cross-arranged. Figs. 3-5 show the diagrammatic sketches of 
the core of three-layered sandwich panel. The basic lengths of the units are the same, which means $H_{0}=\sqrt{3} N_{h 1} l_{h}=N_{t 1} l_{t}$ and $L_{0}=\sqrt{3} N_{h 2} l_{h=} N_{t 2} l_{t}$, and the lengths of unit of $\mathrm{RH}$ are set that $H_{0}=30 \mathrm{~mm}$ and $L_{0}=15 \mathrm{~mm}$. Unless otherwise specified, the $N_{h_{1}}=8, N_{h_{2}}=4, N_{t 1}=12, N_{t 2}=6, l_{t}=2.5 \mathrm{~mm}, H_{c}=25.98 \mathrm{~mm}$ are adopted in the FE simulations. All components of models are meshed using quad Belytschko-Tsay shell elements with five integration points in thickness. A convergence test is conducted to determine the mesh size to ensure the accuracy of the computations, and $1.25 \mathrm{~mm} \times 1.25 \mathrm{~mm}$ is adopted as the element size. The FEM model of RH, RHH and RHT sandwich panels contains 272250 elements, 502200 elements and 942209 elements, respectively. The aluminum alloy AA6063-T6 is adopted as the base material for the face sheets with a thickness of $2 \mathrm{~mm}$. The cores and the interlayer sheets are aluminum alloy AA5182, and the thickness of interlayer sheets is set to 0.5 $\mathrm{mm}$.

The material properties of AA6063-T6 are: the density $\rho=2.7 \times 10^{3} \mathrm{~kg} / \mathrm{m}^{3}$, Young's modulus $E=73.0 \mathrm{GPa}$, tangent modulus $G=28.1 \mathrm{GPa}$, initial yield stress $\sigma_{y}=206 \mathrm{MPa}$, the ultimate stress $\sigma_{u}=257 \mathrm{MPa}$ and Poisson's ratio $v=0.3$ [34]. The effect of strain rate is neglected by reason of the weak strain rate sensitivity of aluminum alloys.

In the simulations, an automatic surface-to-surface contact is defined between the rigid body with a constant velocity and the upper face sheet. The *CONSTRAINED_EXTRA_NODES_SET* is used between the fixed rigid body and the lower face sheet in order to simulate the contact of the sandwich panel and the 
test machine. Furthermore, an automatic single-surface contact used to avoid penetration during deformation is considered.

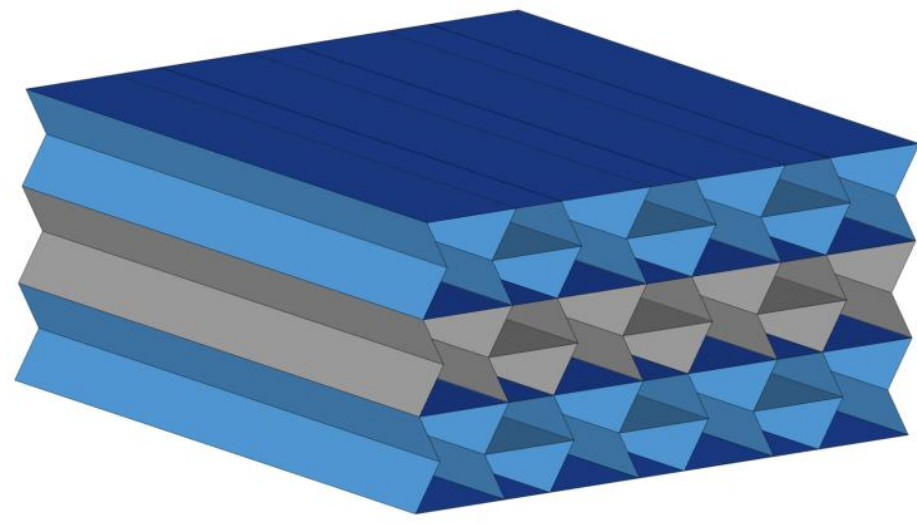

(a) regularly-arranged

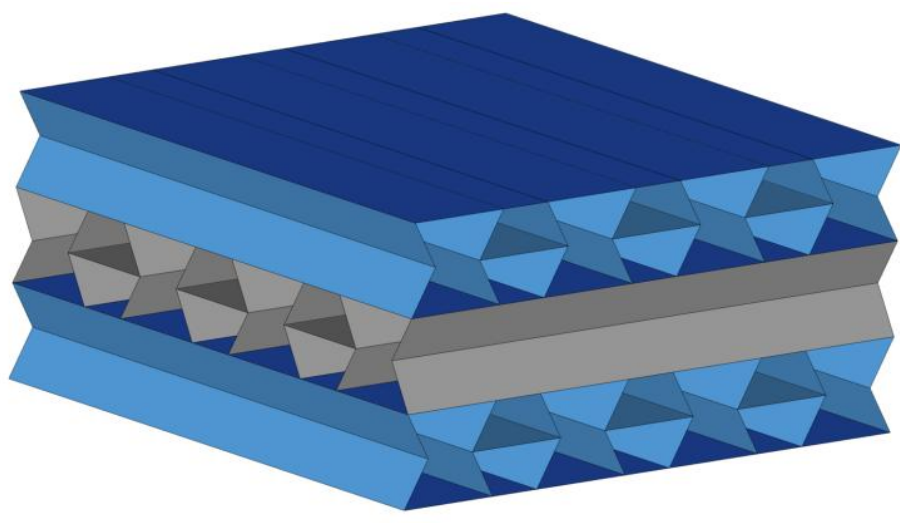

(b) cross-arranged

Fig. 3. Diagrammatic sketches of three-layered RH sandwich panels with different arranged orientations: (a) regularly-arranged; (b) cross-arranged.

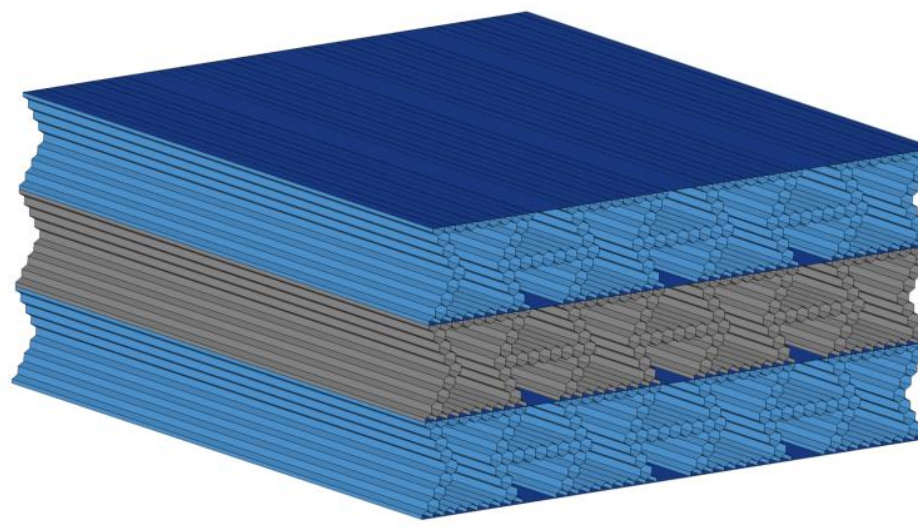

(a) regularly-arranged

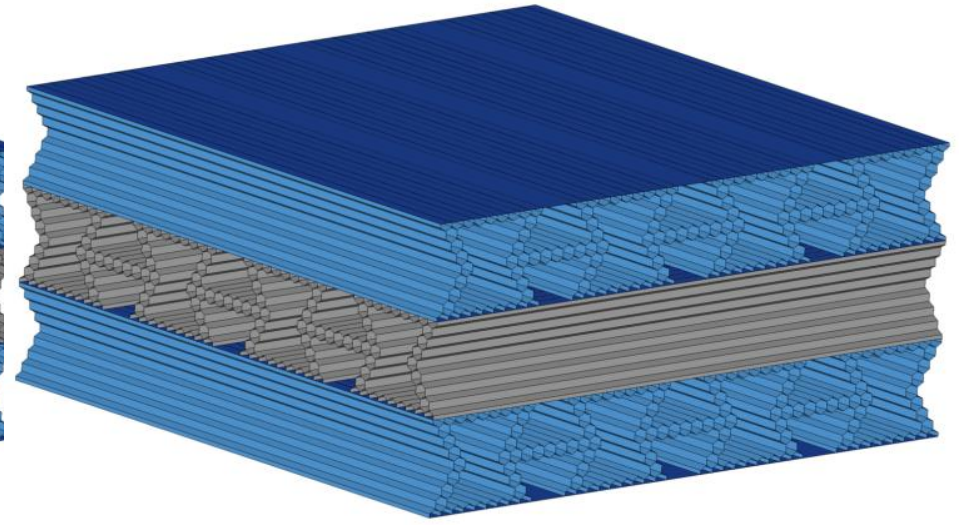

(b) cross-arranged

Fig. 4. Diagrammatic sketches of three-layered RHH sandwich panels with different arranged orientations: (a) regularly-arranged; (b) cross-arranged. 


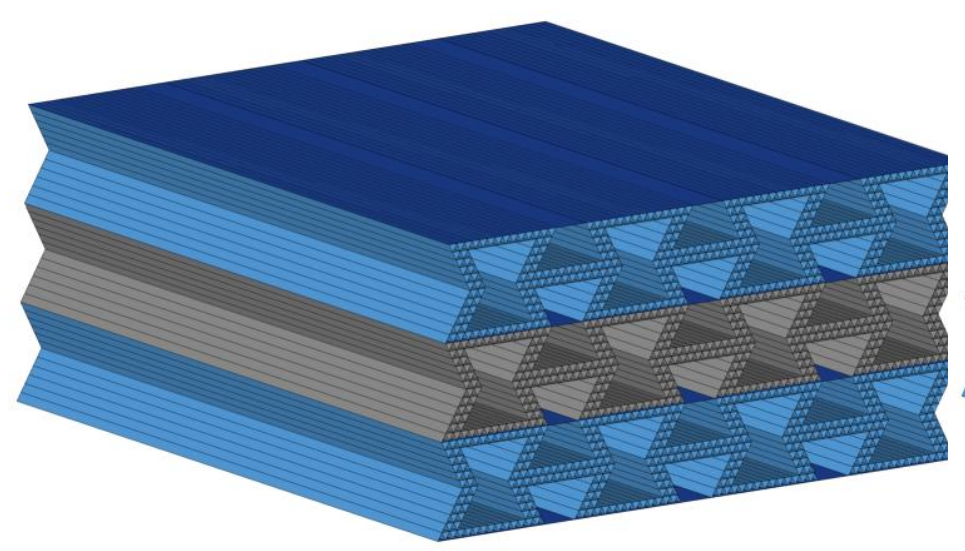

(a) regularly-arranged

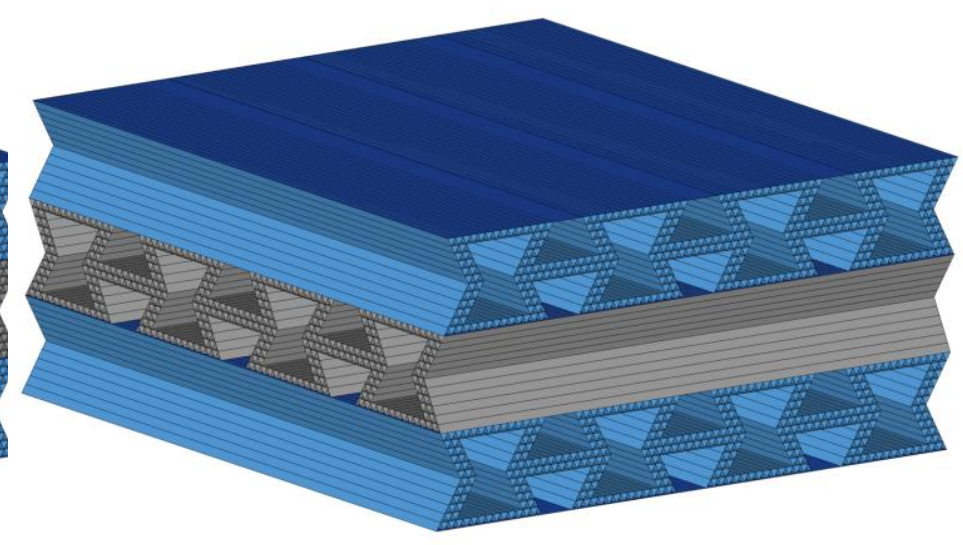

(b) cross-arranged

Fig. 5. Diagrammatic sketches of three-layered RHT sandwich panels with different arranged orientations: (a) regularly-arranged; (b) cross-arranged.

\subsection{Validation of the numerical approach}

In this section, the numerical approach is validated by a series of experiments. The core cell and interlayer sheet of the three-layer regularly-arranged sandwich is modeled with a thickness of $0.2 \mathrm{~mm}$, and the thickness of the face sheets is set to $1 \mathrm{~mm}$ which is the same as the experiments [35]. In order to reduce computation time, a constant speed of $0.5 \mathrm{~m} / \mathrm{s}$ was adopted in the simulation. Fig. 6 presents the deformation of the sandwich panel, and it is seen that deformation of the numerical model is in good agreement with the experiment. Furthermore, the comparison of the force between simulation and the experiment is also made in Fig. 7, and it can be clearly seen that the data form the simulation is very close to the experiment though the results have a slight deviation. Therefore, it can be concluded that the numerical model is proved to be reliable to study the crushing performance of the novel sandwich panels. 


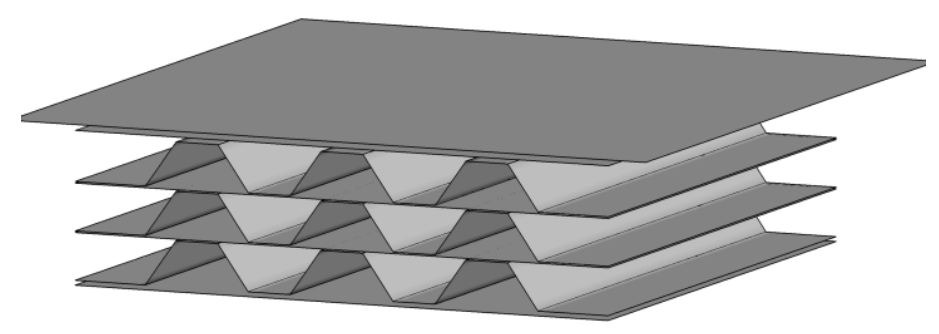

(a)

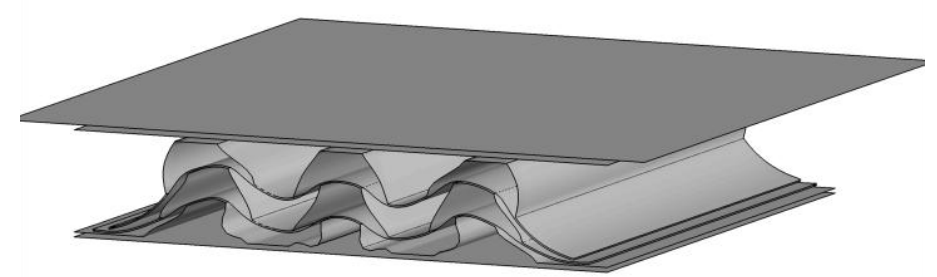

(c)

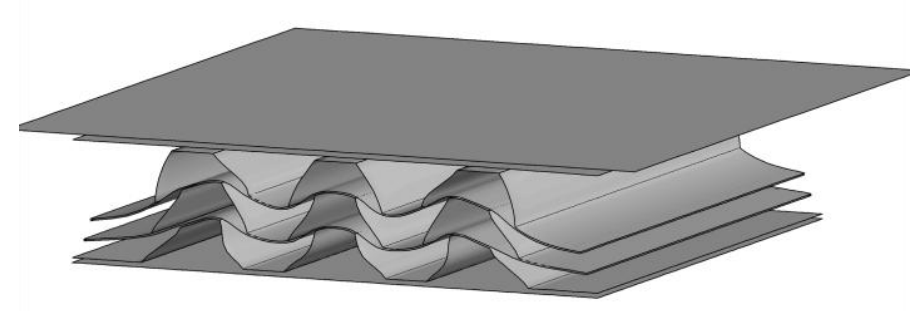

(b)

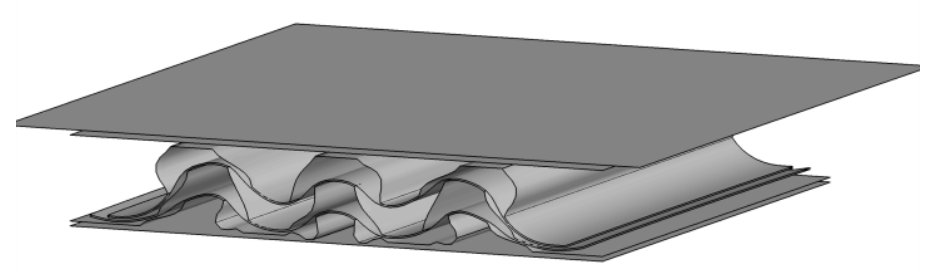

(d)

Fig. 6. Verification of deformation of finite element models compared to the experiment [35]. (a) $d=0 \mathrm{~mm}$. (b) $d=4 \mathrm{~mm}$. (c) $d=6 \mathrm{~mm}$. (d) $d=8 \mathrm{~mm}$.

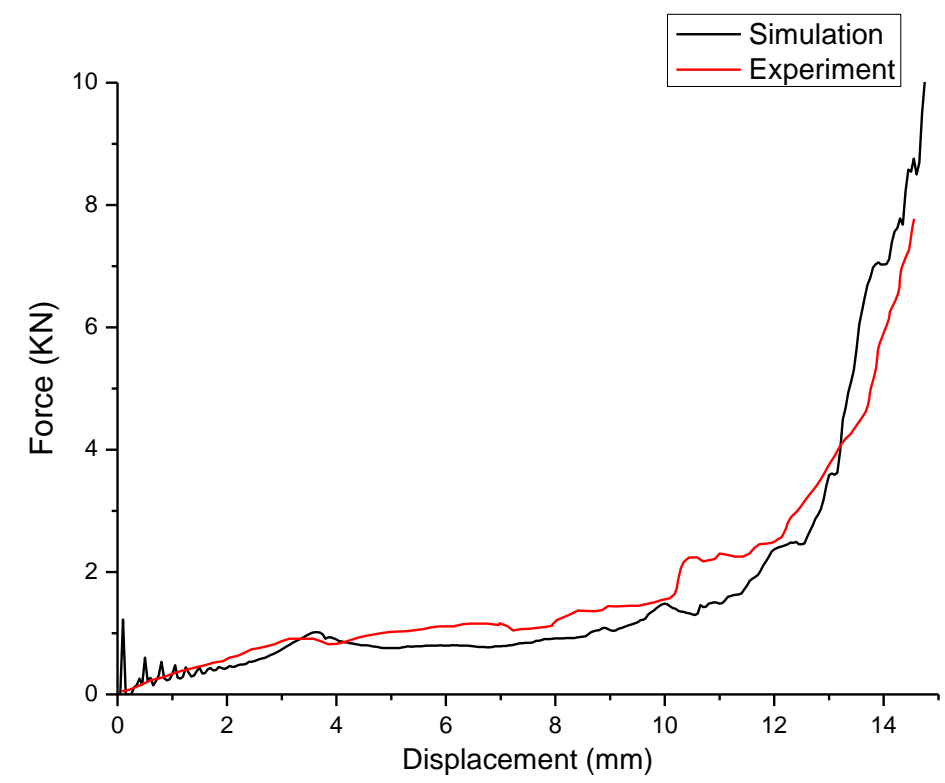

Fig. 7. Comparison of the force obtained from the experiment [35] and the FE analysis.

\section{In plane stiffness of Hierarchical honeycomb}

In this section, we assume all the cell walls of RH, RHH and RHT are Euler 
beams for the sake of the simplicity of the calculation. In other words, we just consider the bending of the cell walls of these structures, which requires that $t_{0} / L_{0}<$ $0.2, t_{0} / H_{0}<0.2, t_{h} / l_{h}<0.2$ and $t_{t} / l_{t}<0.2[36]$.

We define $E_{s}$ as the Young's modulus of the base material, $E_{r 1}$ and $E_{r 2}$ as the Young's modulus of RH $\left(\theta=-30^{\circ}\right)$ in the 1 and 2 directions, $E_{r h 1}$ and $E_{r h 2}$ as the Young's modulus of RHH in the 1 and 2 directions, $E_{r t 1}$ and $E_{r t 2}$ as the Young's modulus of RHT in the 1 and 2 directions, $E_{h}$ as the Young's modulus of isotropic regular hexagon substructures and $E_{t}$ as the Young's modulus of isotropic equilateral triangle substructures. According to [36], we find:

$$
\begin{aligned}
& \frac{E_{r 1}}{E_{s}}=\left(\frac{t_{0}}{l_{0}}\right)^{3} \frac{\cos \theta}{\left(H_{0} / l_{0}+\sin \theta\right) \sin ^{2} \theta} \\
& \frac{E_{r 2}}{E_{s}}=\left(\frac{t_{0}}{l_{0}}\right)^{3} \frac{\left(H_{0} / l_{0}+\sin \theta\right)}{\cos ^{3} \theta} \\
& \frac{E_{h}}{E_{s}}=2.3\left(\frac{t_{h}}{l_{h}}\right)^{3} \\
& \frac{E_{t}}{E_{s}}=\frac{2}{\sqrt{3}} \frac{t_{t}}{l_{t}}
\end{aligned}
$$

Based on the derived formulas for Young's modulus of multifunctional hierarchical honeycomb [37, 38], the Young's modulus of RHH is derived by:

$$
\begin{aligned}
& \frac{E_{r h 1}}{E_{h}}=\left(\frac{T_{1}}{l_{0}}\right)^{3} \frac{\cos \theta}{\left(N_{h 1} / N_{h 2}+\sin \theta\right) \sin ^{2} \theta} \\
& \frac{E_{r h 2}}{E_{h}}=\left(\frac{T_{1}}{l_{0}}\right)^{3} \frac{\left(N_{h 1} / N_{h 2}+\sin \theta\right)}{\cos ^{3} \theta}
\end{aligned}
$$




$$
\begin{aligned}
& \frac{E_{r h 1}}{E_{s}}=2.3\left(\frac{T_{1}}{l_{0}} \frac{t_{h}}{l_{h}}\right)^{3} \frac{\cos \theta}{\left(N_{h 1} / N_{h 2}+\sin \theta\right) \sin ^{2} \theta} \\
& \frac{E_{r h 2}}{E_{s}}=2.3\left(\frac{T_{1}}{l_{0}} \frac{t_{h}}{l_{h}}\right)^{3} \frac{\left(N_{h 1} / N_{h 2}+\sin \theta\right)}{\cos ^{3} \theta}
\end{aligned}
$$

For RHT, the Young's modulus is obtained as:

$$
\begin{aligned}
& \frac{E_{r t 1}}{E_{t}}=\left(\frac{T_{2}}{l_{0}}\right)^{3} \frac{\cos \theta}{\left(N_{t 1} / N_{t 2}+\sin \theta\right) \sin ^{2} \theta} \\
& \frac{E_{r t 2}}{E_{t}}=\left(\frac{T_{2}}{l_{0}}\right)^{3} \frac{\left(N_{t 1} / N_{t 2}+\sin \theta\right)}{\cos ^{3} \theta} \\
& \frac{E_{r t 1}}{E_{s}}=\frac{2}{\sqrt{3}} \frac{t_{t}}{l_{t}}\left(\frac{T_{2}}{l_{0}}\right)^{3} \frac{\cos \theta}{\left(N_{t 1} / N_{t 2}+\sin \theta\right) \sin ^{2} \theta} \\
& \frac{E_{r t 2}}{E_{s}}=\frac{2}{\sqrt{3}} \frac{t_{t}}{l_{t}}\left(\frac{T_{2}}{l_{0}}\right)^{3} \frac{\left.N_{t 1} / N_{t 2}+\sin \theta\right)}{\cos ^{3} \theta}
\end{aligned}
$$

It should be noted that Eqs. (7)-(18) are only valid for infinite honeycombs. Combining Eqs. (9), (10), (13), (14), (17) and Eq. (18), the relative Young's modulus in the 1 and 2 directions can be obtained as:

$$
\begin{aligned}
& \frac{E_{r h 1}}{E_{r 1}}=\frac{E_{r h 2}}{E_{r 2}}=2.3\left(\frac{T_{1}}{t_{0}} \frac{t_{h}}{l_{h}}\right)^{3} \\
& \frac{E_{r t 1}}{E_{r 1}}=\frac{E_{r t 2}}{E_{r 2}}=\frac{2}{\sqrt{3}} \frac{t_{t}}{l_{t}}\left(\frac{T_{2}}{t_{0}}\right)^{3}
\end{aligned}
$$

As shown in Fig. 8, for the given configurations of hierarchical honeycombs which are described in section 2.3 , it can be seen that the introduction of hierarchy can greatly improve the Young's modulus of a honeycomb. Compared with RH, the 
enhancements of the relative in-plane stiffness of the RHH and RHT could be 1.3 times and 6.7 times respectively at relative density of 0.1 . Interestingly, the lower the relative density is, the more in-plane stiffness RHT increases. By contrast, the increased in-plane stiffness of RHH remains unchanged under different relative densities.

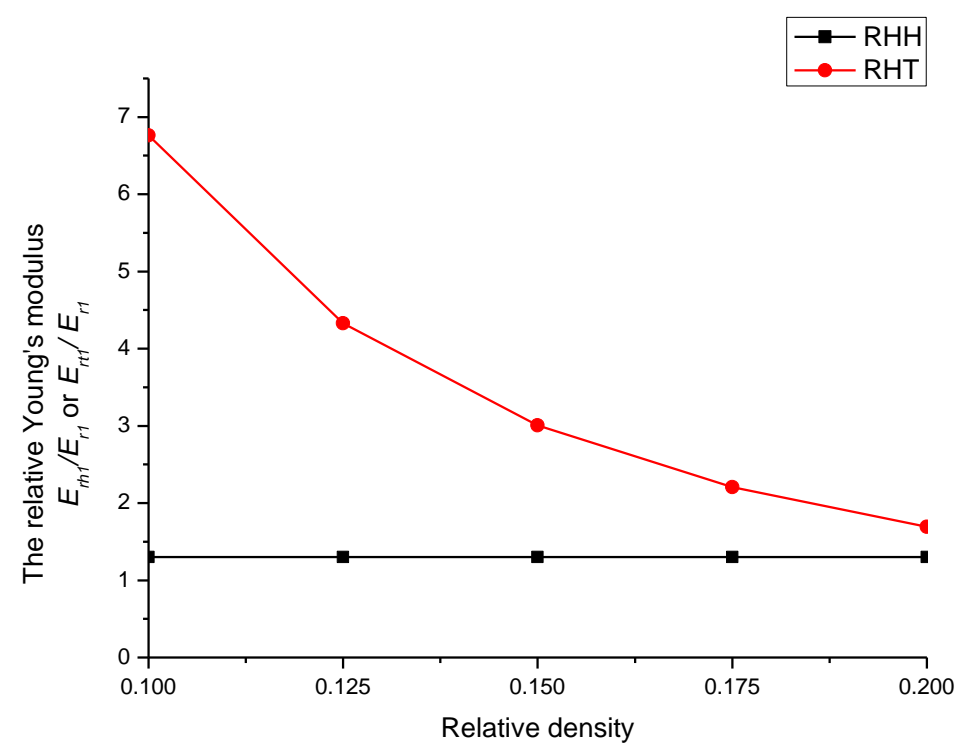

Fig. 8. The relative Young's modulus $E_{\mathrm{rh} 1} / E_{\mathrm{r} 1}$ or $E_{\mathrm{rt} 1} / E_{\mathrm{r} 1}$ of re-entrant hierarchical honeycombs for different relative densities.

\section{Results and discussion}

In this section, constant velocities are applied to the moving rigid body which is used to investigate the crashworthiness of these sandwich structures. The moving rigid body is a square plate with a side of length $268.6 \mathrm{~mm}$, and the mass of the plate is $1.96 \mathrm{~kg}$ as shown in Fig. 9. The lower plate is fixed with all the degrees of freedom of the nodes. In order to facilitate comparison, the masses of all cores of models are set equal. The relative density of honeycomb cores is significantly affected by the thickness of honeycomb cores. The graded honeycomb cores can be made by varying 
the thickness of the honeycomb cores. As mentioned above, the relative densities of $10 \%, 15 \%$ and $20 \%$ are adopted to generate graded cores respectively corresponding to small(S), medium (M) and large (L). The thickness and the weight of the honeycomb cores with different relative densities are given in Table 1. Because the cores are made of three layers (upper, middle and lower), there are six different graded configurations of the sandwich panels for keeping the total mass of the sandwich structure unchanged. Both regularly-arranged (R) and cross-arranged (C) sandwich panels (RH, RHH and RHT) have two typical graded configurations: R-SML, R-LMS, C-SML, C-LMS and an ungraded configuration R-MMM, C-MMM.

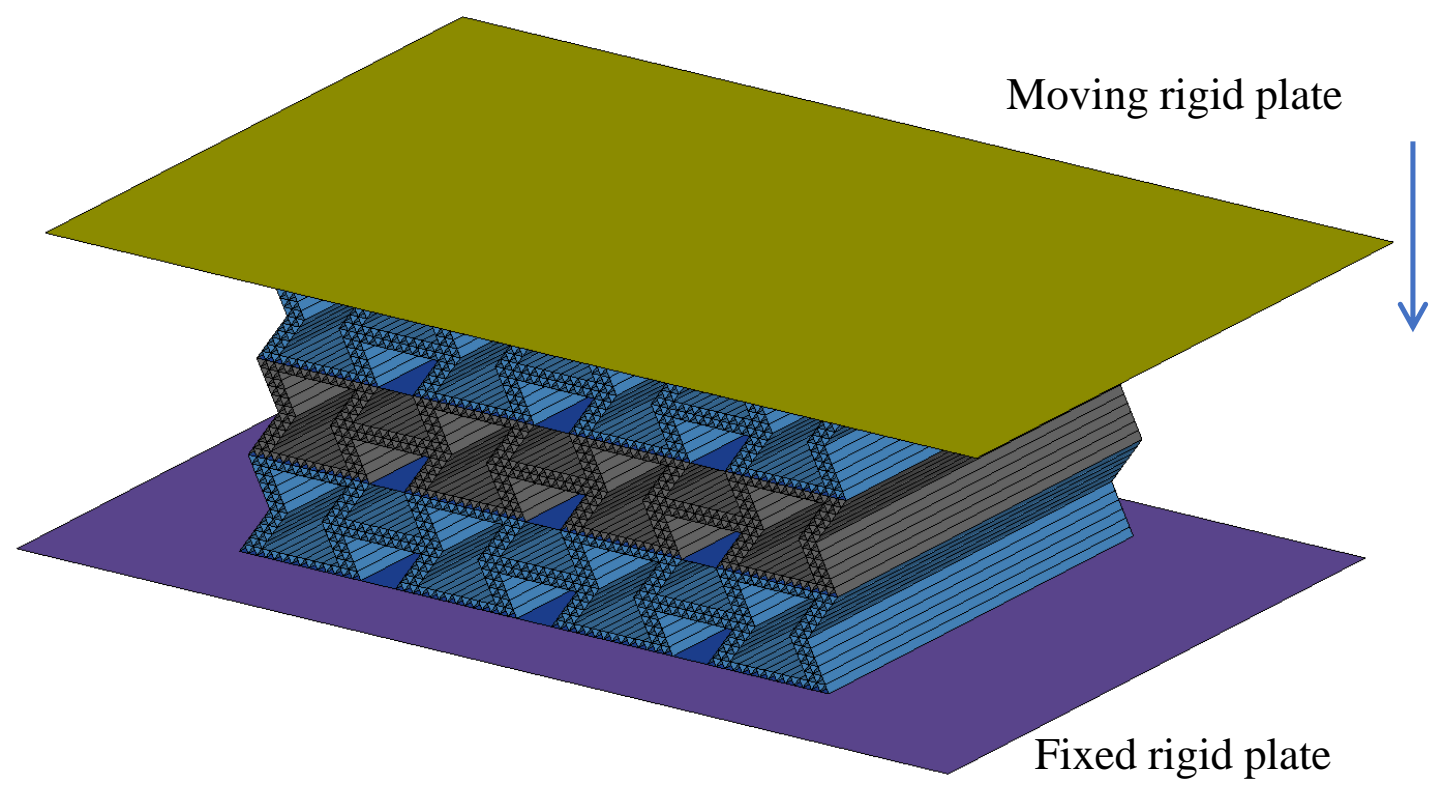

Fig. 9. The configuration of sandwich panels under dynamic crushing condition.

Table 1

Thickness and weight of layers of graded honeycomb cores at different relative densities

\begin{tabular}{clccc}
\hline & & RH & RHH & RHT \\
\hline Relative density & Thickness (mm) & 0.9742 & 0.2739 & 0.1173 \\
of 0.10 & Weight $(\mathrm{kg})$ & 0.1432 & 0.1432 & 0.1432 \\
Relative density & Thickness (mm) & 1.4614 & 0.4109 & 0.1759 \\
of 0.15 & Weight $(\mathrm{kg})$ & 0.2148 & 0.2148 & 0.2148 \\
Relative density & Thickness $(\mathrm{mm})$ & 1.9485 & 0.5479 & 0.2346 \\
of 0.20 & Weight $(\mathrm{kg})$ & 0.2864 & 0.2864 & 0.2864 \\
\hline
\end{tabular}




\subsection{Energy absorption capability}

Fig. 10 presents an overview of energy absorption of sandwich panels with different configuration at a crushing strain of $88.78 \%$ when the impact velocity is $1 \mathrm{~m} / \mathrm{s}$. From the results in Fig. 10, it is apparent that the energy absorption capacity of re-entrant hierarchical sandwich panels is much better than regular re-entrant sandwich panel. The RHH sandwich panels absorb more than twice energy than RH sandwich panels, and the RHT sandwich panels absorb almost three times as much energy as RH sandwich panels. Here, the MCF and SEA are employed to evaluate the impact performance of sandwich panels. It should be mentioned that the higher the value of MCF and SEA, the higher the energy absorption capacity. The comparison of MCF of different sandwich panels with configuration of R-MMM is presented in Fig. 11. It is clear that RHT sandwich panel has the largest value of MCF in the compression process. The MCF of RHH sandwich panel is smaller than RH sandwich panel before densification strain, but much higher in the later stage. It is worth noting that the value of MCF of RHT sandwich panel is $298.2 \%$ higher than the value of RH, and the RHH sandwich panel is $208.2 \%$ higher than RH when the crushing distance is 70mm. Fig. 12 compares the results of SEA, and the RHT sandwich panel still has the highest SEA capacity among these sandwich panels. The RHT sandwich panel improves SEA by $269.1 \%$, and RHH sandwich panel improves SEA by $203.1 \%$ above $\mathrm{RH}$ sandwich panel at a displacement of $70 \mathrm{~mm}$. Based on previous results, it can be concluded that the introduction of hierarchy into sandwich panel can greatly improve the energy absorption capacity. 


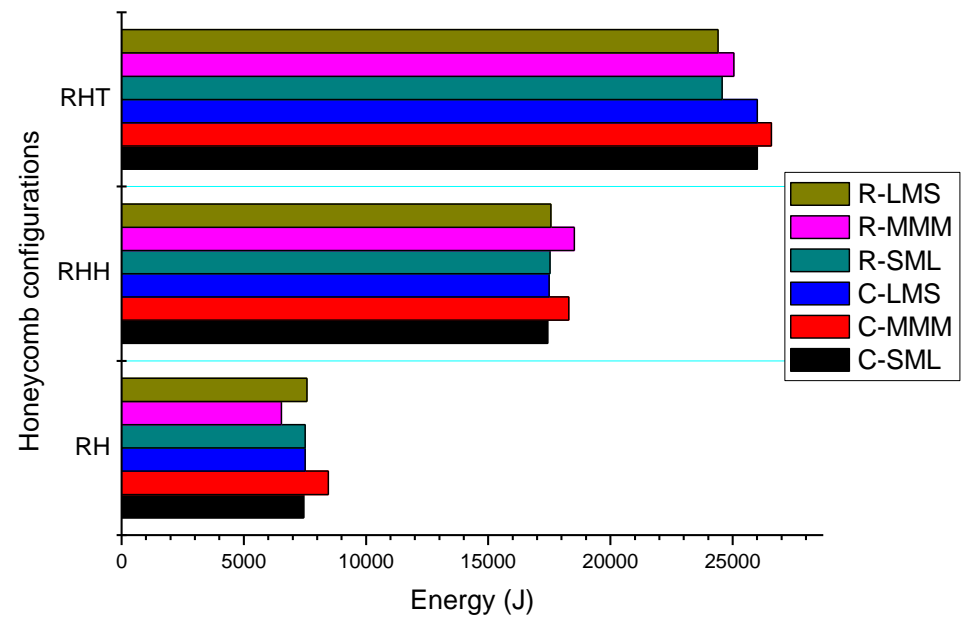

Fig. 10. Energy absorption of different configurations of sandwich panels.

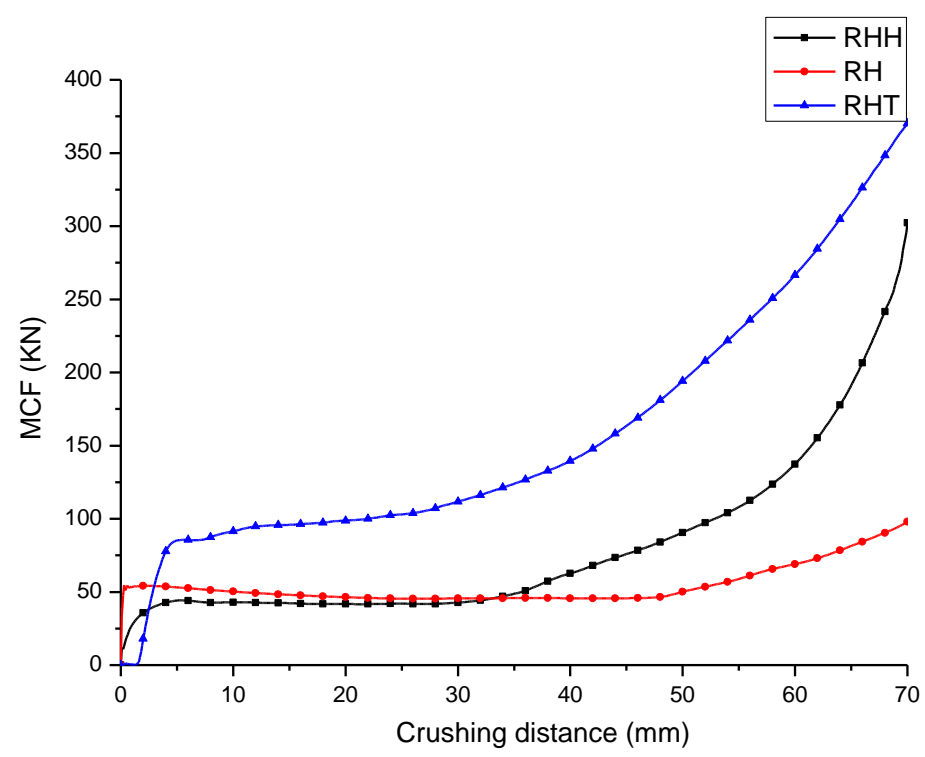

Fig. 11. Comparsion of MCF of different sandwich panels for the configuration of R-MMM. 


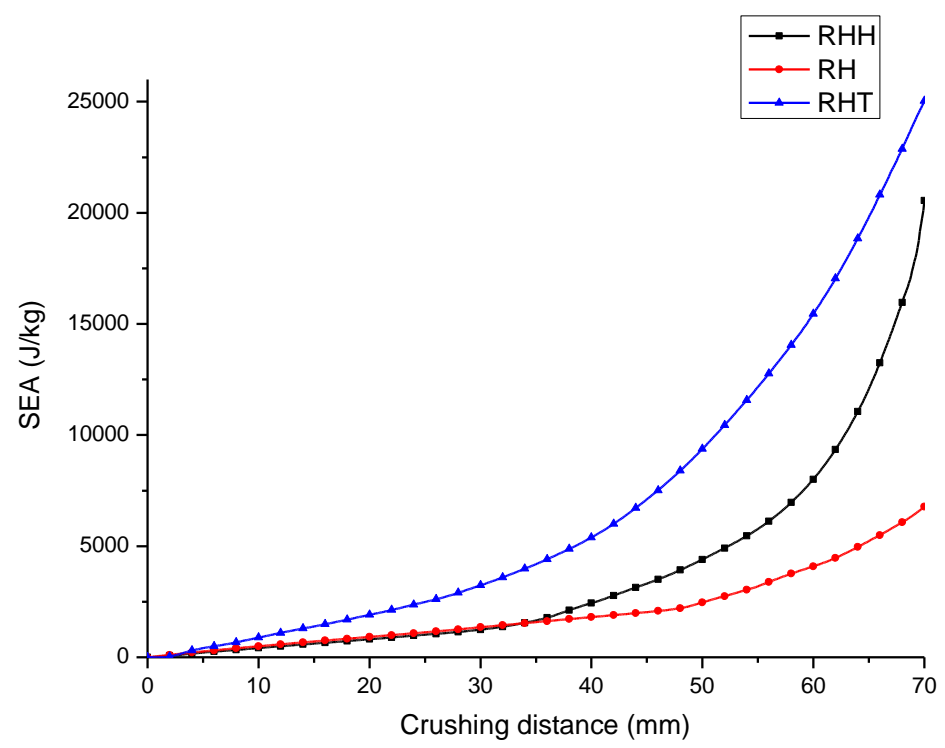

Fig. 12. Comparsion of SEA of different sandwich panels for the configuration of R-MMM.

In order to study the mechanism of the huge increase in energy absorption of hierarchical sandwich panels, the stress diagrams of sandwich panels are provided in Fig. 13 to visualize the stress distribution of the sandwich panels. Hierarchical re-entrant sandwich panels have a lot of substructures. In contrast, the traditional sandwich panel only has basic structure, which means that the thickness of RH sandwich panel is much thicker than hierarchical sandwich panel in order to keep the same mass of all honeycomb cores. As a consequence, the edges of hierarchical sandwich panels become more transmutable and create more folds during the collision, and thus conduce to excellent energy absorption ability. As can be seen from Fig. 13, the folds in the white circles can been observed, and RHT has the most plastic hinges which means the highest energy absorption capability. 


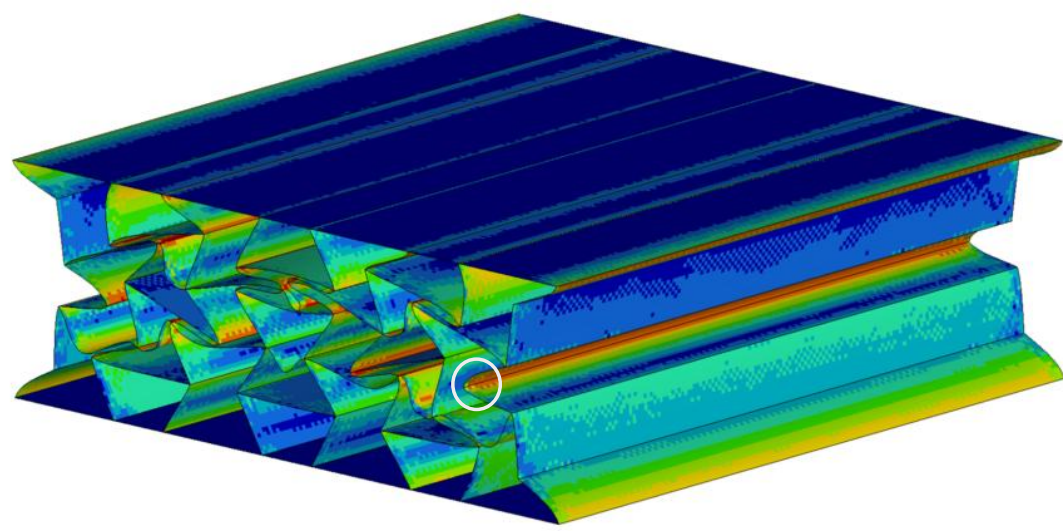

(b)

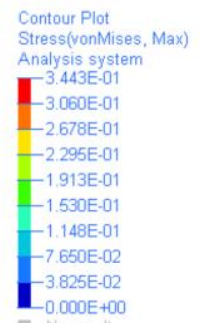

Max $=3.443 \mathrm{E}-01$

IENT_SHELL 11884478

ELEMENT SHELL 12759215

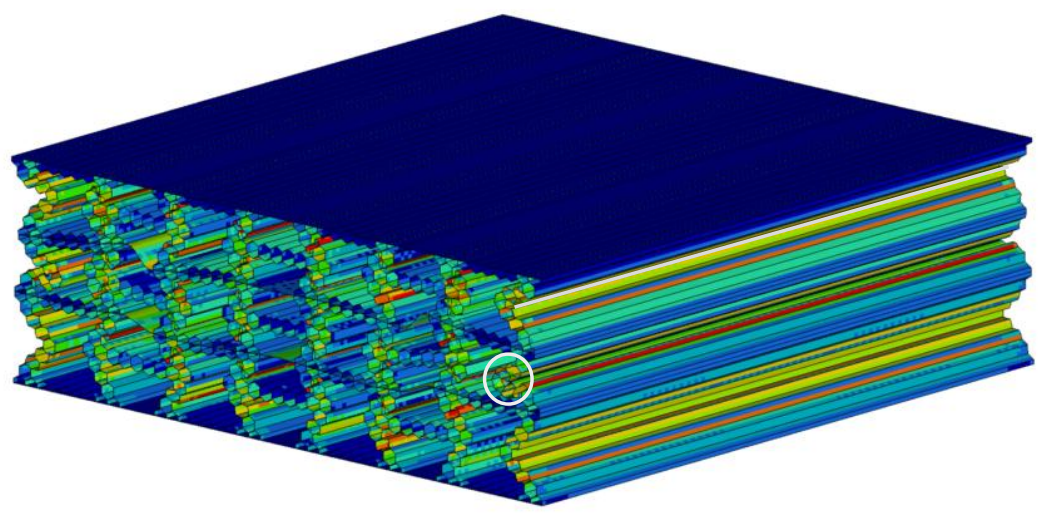

(c)
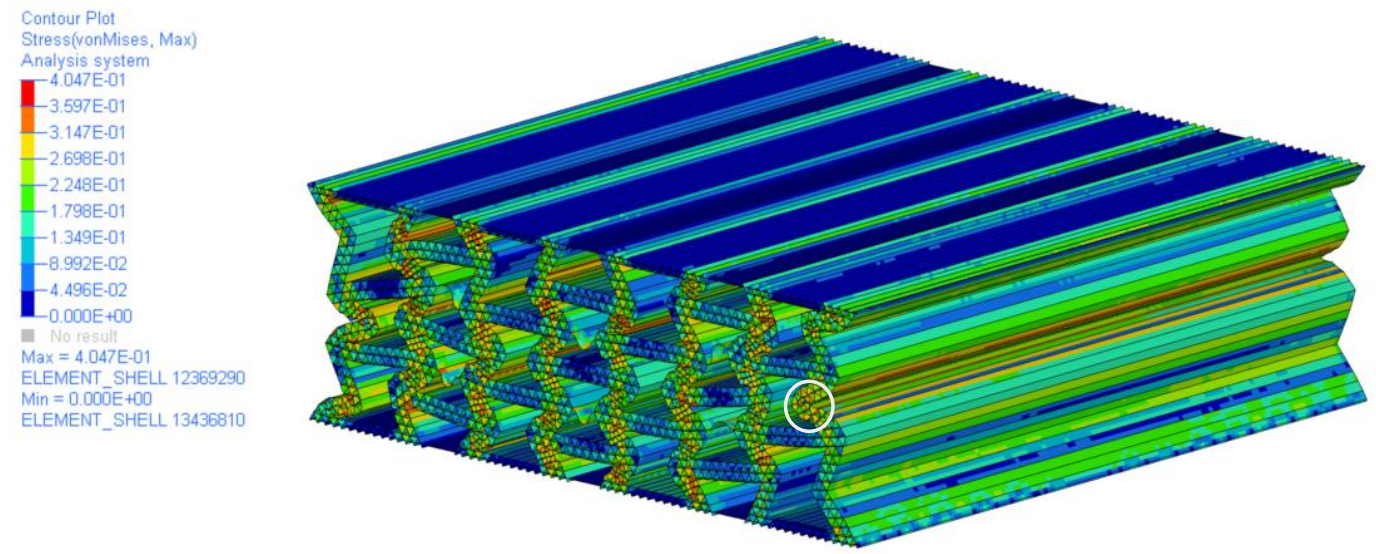

Fig. 13. Stress diagrams of different sandwich panels at a strain of 0.28:(a) RH; (b)RHH; (c)RHT.

\subsection{Response of the graded hierarchical honeycomb cores}

In this section, gradient is introduced to hierarchical sandwich panels. Graded

hierarchical sandwich panels are proposed by different graded cores in order to give a 
thorough research of the graded effect on deformation modes, crushing forces and energy absorption abilities.

The relative density of sandwich core obviously changes with the changing of the thickness. As described before, for RHH sandwich panel, the thicknesses of graded cores are $0.2739 \mathrm{~mm}, 0.4109 \mathrm{~mm}$ and $0.5479 \mathrm{~mm}$ respectively for the honeycomb cores with $\mathrm{S}, \mathrm{M}$ and $\mathrm{L}$, and thicknesses of $0.1173 \mathrm{~mm}, 0.1759 \mathrm{~mm}$ and $0.2346 \mathrm{~mm}$ are used to generate gradient for RHT sandwich panel. The regularly-arranged hierarchical sandwich panel is used to discuss the effect of gradient in this section.

\subsubsection{Effect of gradient distribution on deformation modes of sandwich panels.}

The deformation modes of hierarchical sandwich panels with different configurations are discussed here. As shown in Fig. 14(a) and (b), the deformation modes of graded RHT hierarchical sandwich panels R-SML and R-LMS can be divided into three stages. It can be observed from the figure at crushing distance of $8 \mathrm{~mm}$ that the crushing deformations only occur in S-core, while M-core and L-core basically remain unchanged, which can be summarized as the first stage. As the crushing progress, the S-core has been compressed for a distance; the M-core starts to undergo obvious crushing deformation, which can be regarded as the second stage. The third stage, the L-core begins to deform, and the M-core and S-core continue to collapse. Different from the graded hierarchical sandwich panel, the M-cores of ungraded RHT sandwich panel deforms almost simultaneously, which can be seen in 
Fig. 14(c).

Similar to the RHT hierarchical sandwich panels, the RHH hierarchical sandwich panels exhibit the same deformation characteristics as shown in Fig. 15. The cores of two graded RHH sandwich panels deform separately in the early stage, while the cores of ungraded RHT deform together from the beginning to the end. By comparison of the deformation modes in graded and ungraded hierarchical sandwich panels, it can be summarized that gradient can have a great influence on the deformation modes of hierarchical sandwich panels.

(a)
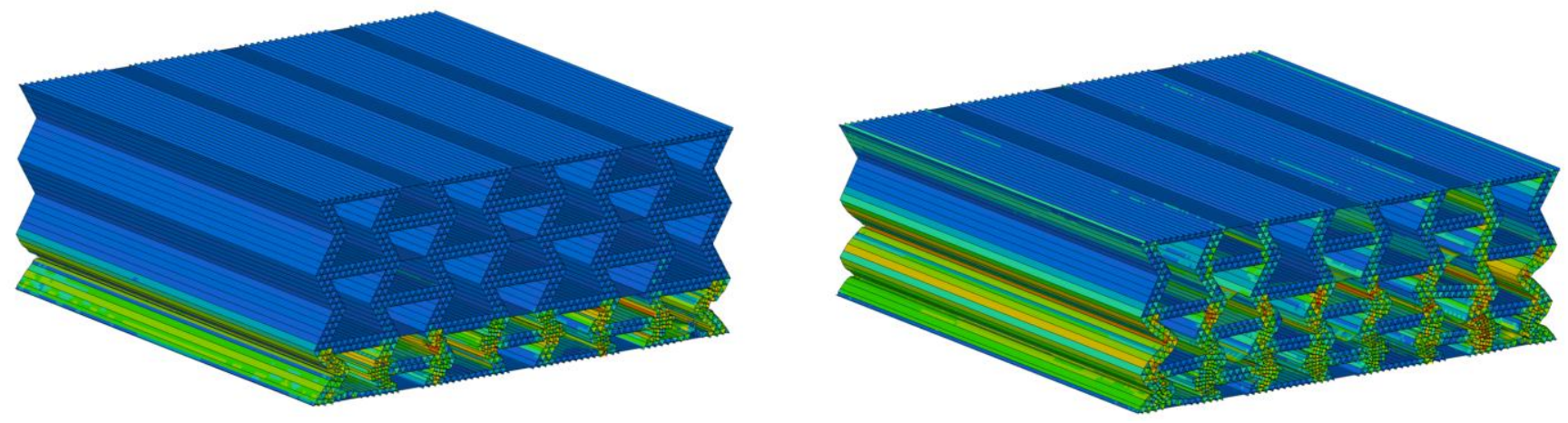

(b)
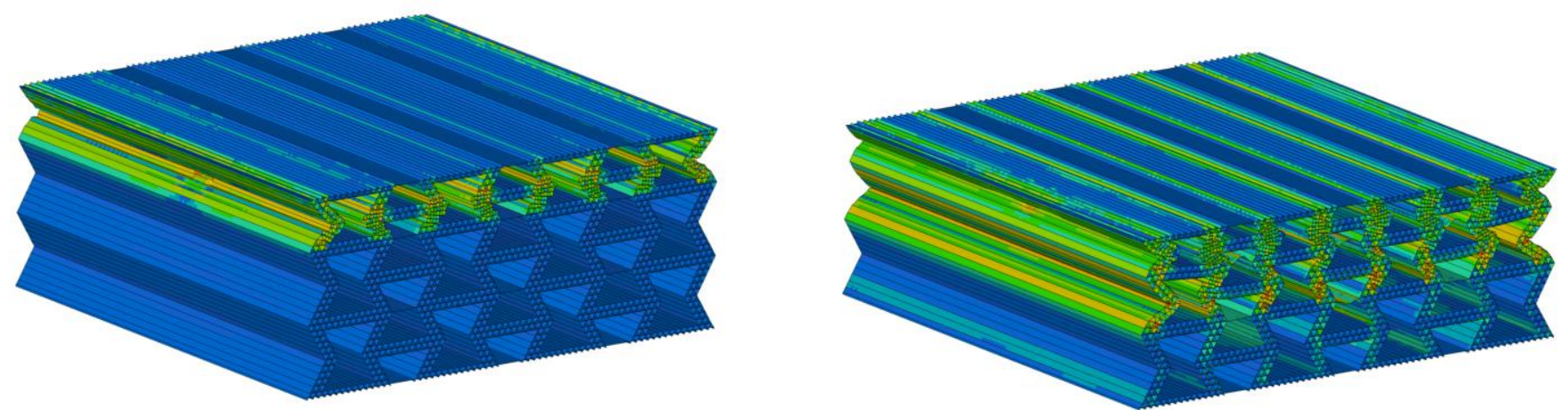

(c)

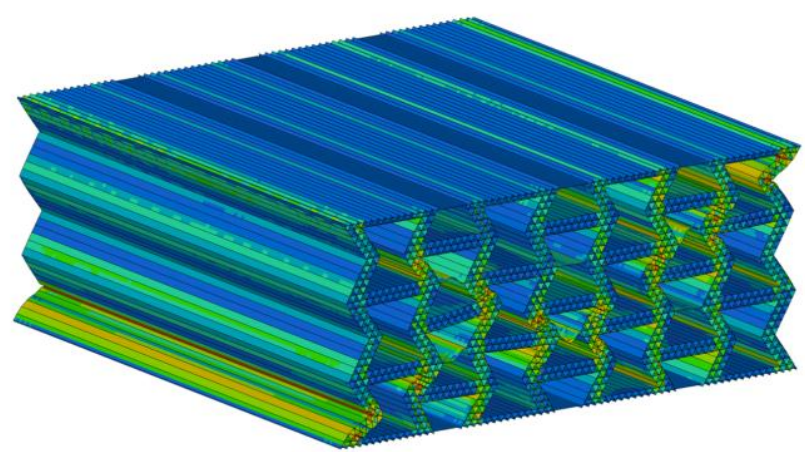

$8 \mathrm{~mm}$

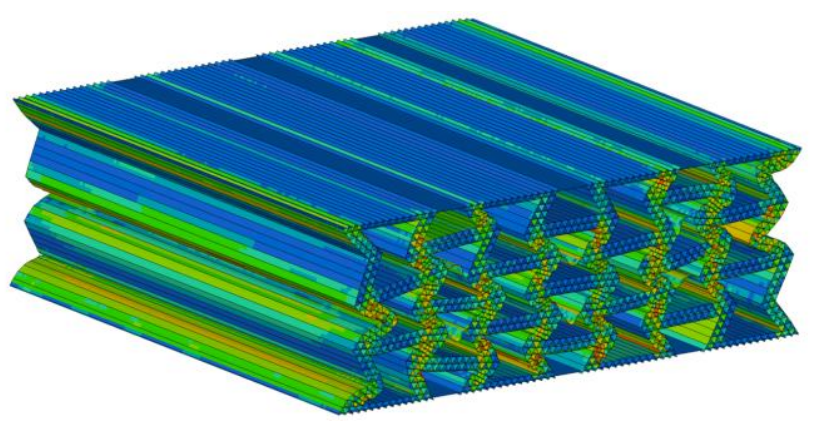

$20 \mathrm{~mm}$ 
Fig. 14. Comparison of stress deformation processes of RHT hierarchical sandwich panels with graded cores: (a) R-LMS, (b) R-SML, (c) R-MMM

(a)
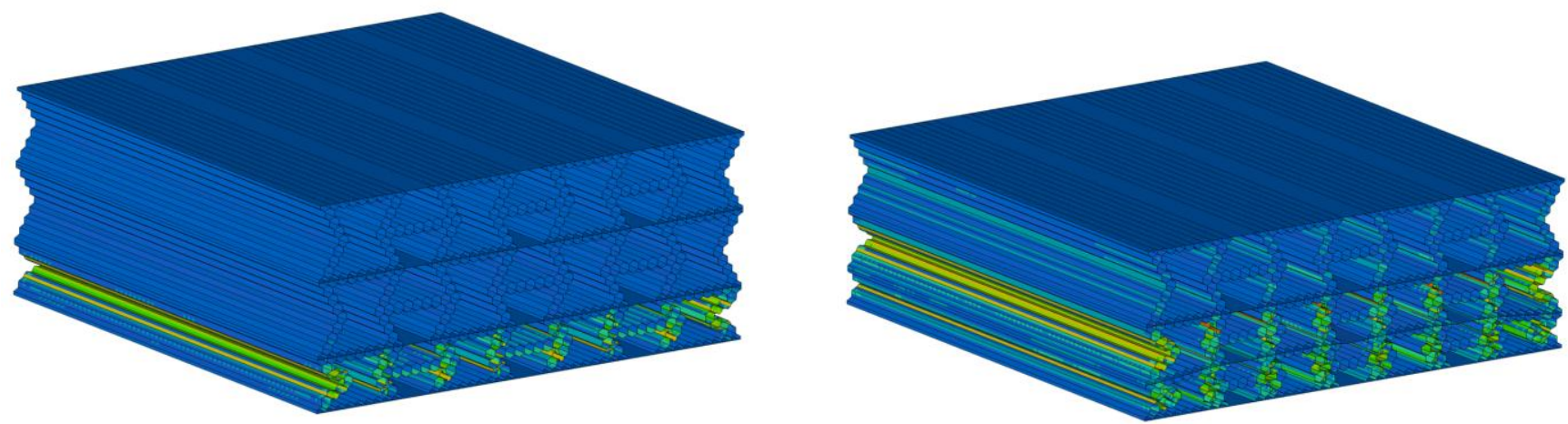

(b)
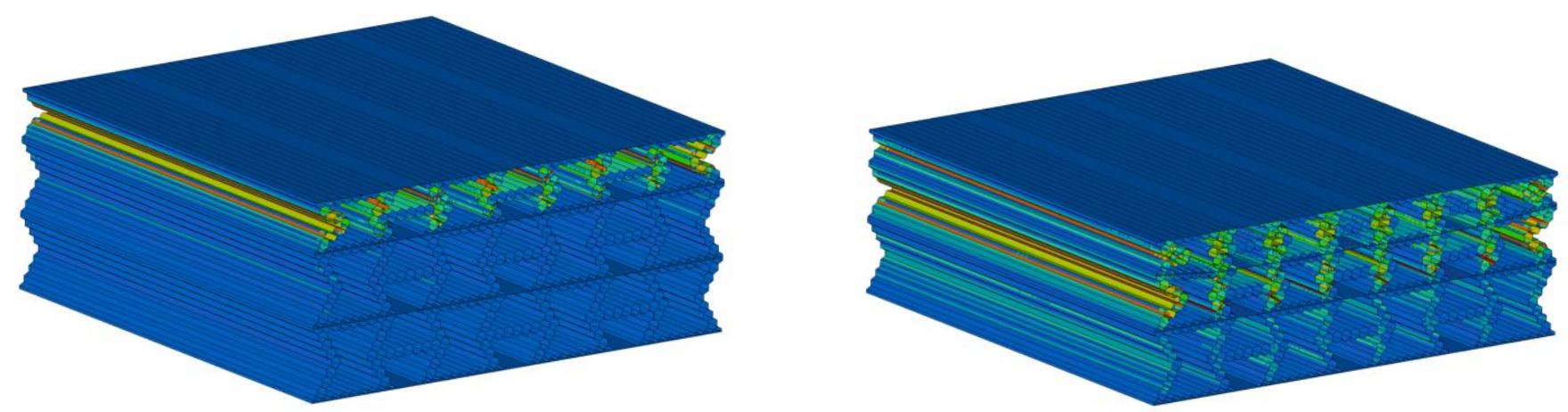

(c)

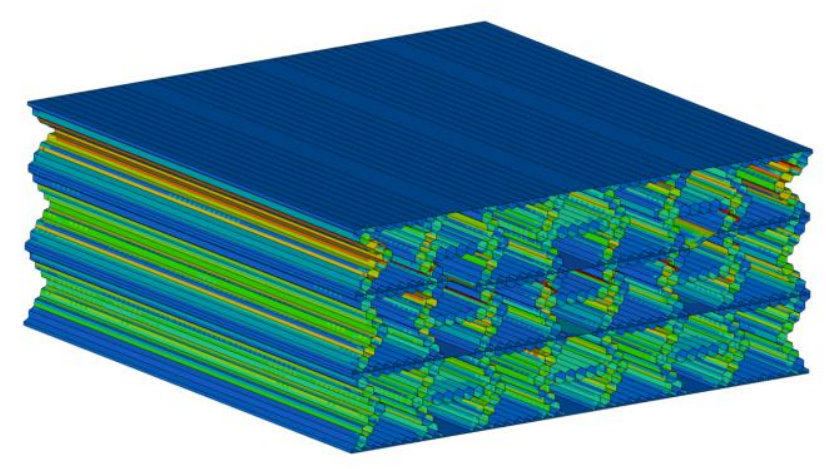

$8 \mathrm{~mm}$

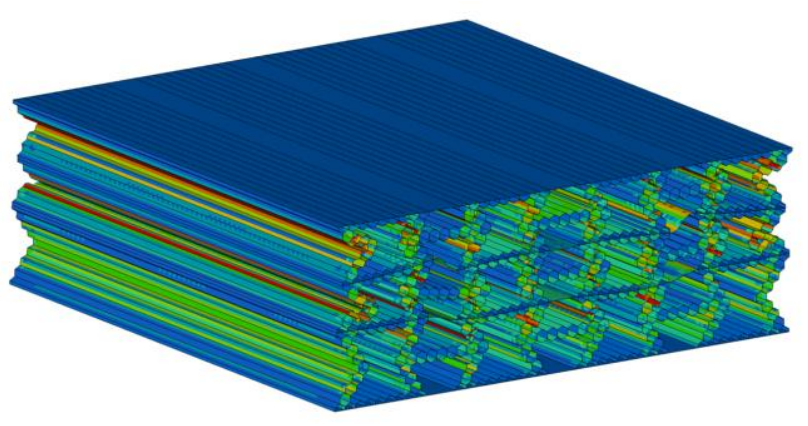

$20 \mathrm{~mm}$

Fig. 15. Comparison of stress deformation processes of RHH hierarchical sandwich panels with graded cores:

(a) R-LMS, (b) R-SML, (c) R-MMM

\subsubsection{Effect of gradient distribution on crushing force}

The force-distance curves of RHT sandwich panel with different graded cores are compared in Fig. 16. The curves of two graded hierarchical sandwich panels R-LMS and R-SML are basically the same. The most remarkable observation to emerge from the comparison is that the two graded sandwich panels have three 
crushing stress plateaus while the ungraded sandwich panels only has one crushing stress plateau. Corresponding to the deformation processes in Fig. 14, the first stress plateau of graded hierarchical sandwich panels appears when the S-core starts to collapse while the M-core and L-core almost remain unchanged. As the S-core is gradually densified, the crushing force starts to increase rapidly. Then, the M-core starts to collapse, which results in the second stress plateau, while the L-core still remains constant. Similarly, as the crushing progresses, the L-core is crushed, and the third stress plateau emerges. When all three cores are ready to further compact, the whole sandwich panel starts to enter densification, and the force increases dramatically. Because of the same thickness of three cores, the three cores of ungraded hierarchical sandwich panels almost collapse at the same time. Interesting, the only stress plateau of ungraded sandwich panel is much wider than the graded sandwich panels, which can be explained that the cores of ungraded sandwich panel collapse together while the cores of graded sandwich panels crumple separately.

Similarly, as shown in Fig. 17, the graded RHH sandwich panels exhibit almost the same characteristics as the graded RHT sandwich panels, and the graded RHH sandwich panels also have three crushing stress plateau and the ungraded RHH sandwich panel still has one crushing stress plateau. Each of the stress plateau emerges accompanied with the collapse of new cores of graded hierarchical sandwich panels.

Combining Fig. 16 and Fig. 17, it should be emphasized that the order of the graded hierarchical cores would not affect the tendency of crushing stress. 
Furthermore, as shown in Table 2, the graded hierarchical sandwich panels can significantly reduce the initial peak force. The configuration of LMS and SML of regularly-arranged RHT sandwich panels can reduce the initial peak force by $35.1 \%$ and $36.6 \%$ respectively. Compared with ungraded regularly-arranged RHH sandwich panel, the R-SML and R-LMS configuration can reduce initial peak force by $56.5 \%$ and $38.0 \%$ respectively. Additionally, in terms of the effects of the gradients, it has almost the same effect on cross-arranged sandwich panels and regular-arranged sandwich panels.

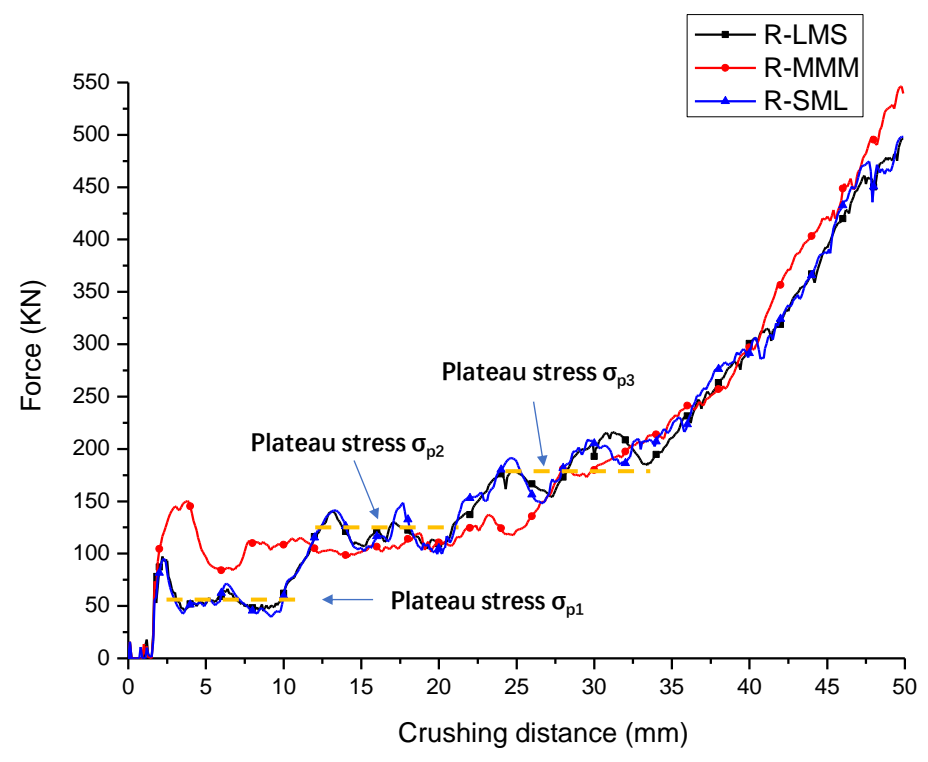

Fig. 16. Contact force-distance curves of RHT sandwich panels with graded cores. 


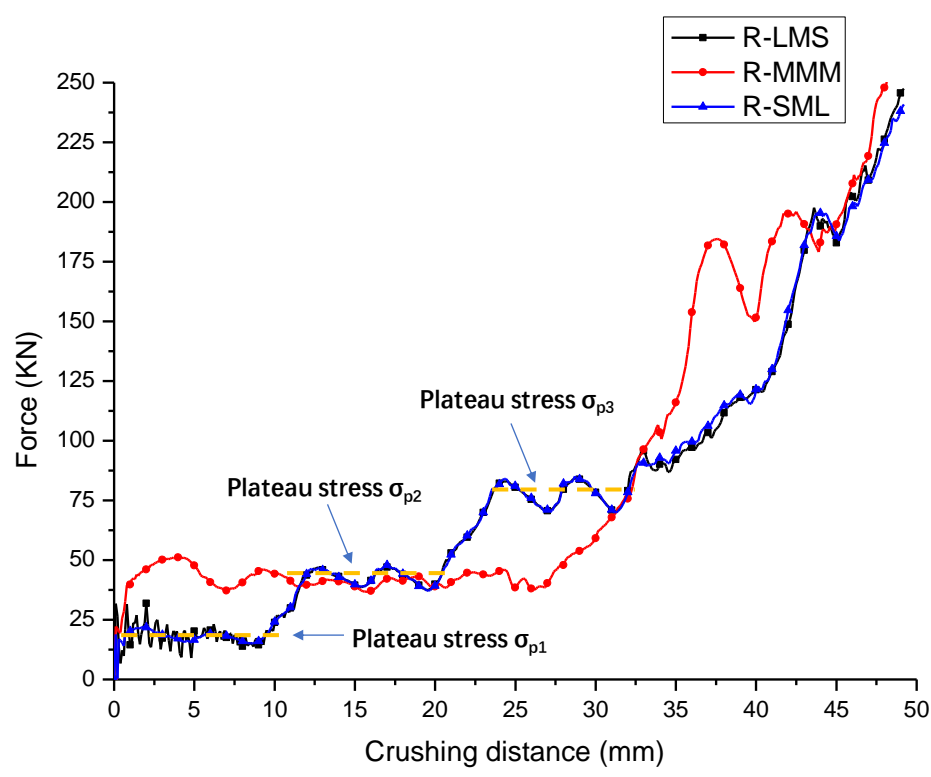

Fig. 17. Contact force-distance curves of RHH sandwich panels with graded cores.

Table 2

Initial peak force of different graded sandwich panels (KN)

\begin{tabular}{cccc}
\hline $\begin{array}{c}\text { Regularly-arranged RHT } \\
\text { sandwich panel }\end{array}$ & 97.4 & MMM & SML \\
$\begin{array}{c}\text { Regularly-arranged RHH } \\
\text { sandwich panel } \\
\text { Cross-arranged RHT } \\
\text { sandwich panel } \\
\text { Cross-arranged RHH } \\
\text { sandwich panel }\end{array}$ & 31.6 & 150.2 & 95.1 \\
\hline
\end{tabular}

\subsubsection{Effect of gradient distribution on energy absorption}

Energy absorption-strain curves of different graded configurations with different impact velocities are compared in Fig. 18-20. Obviously, the gradient distribution has a significant effect on the energy absorption ability. As presented in Fig.18, Under the quasi-static condition of $1 \mathrm{~m} / \mathrm{s}$ velocity, the ungraded configuration of hierarchical sandwich panels absorb the maximum energy. Furthermore, there is a very small difference of energy absorption between the configurations of R-LMS and 
R-SML. In the low impact speed of $10 \mathrm{~m} / \mathrm{s}$ case, it can be found that the R-LMS hierarchical sandwich panels absorb the maximum energy while the R-SML panels absorb the minimum energy in the first phase. After that, the ungraded sandwich panels exhibit better energy absorption ability than graded specimens. When the impact velocity is $50 \mathrm{~m} / \mathrm{s}$, the R-LMS panels still have the highest energy absorption ability. What is interesting in these figures is that the configuration of R-SML always absorbs the minimum energy compared with other configurations.

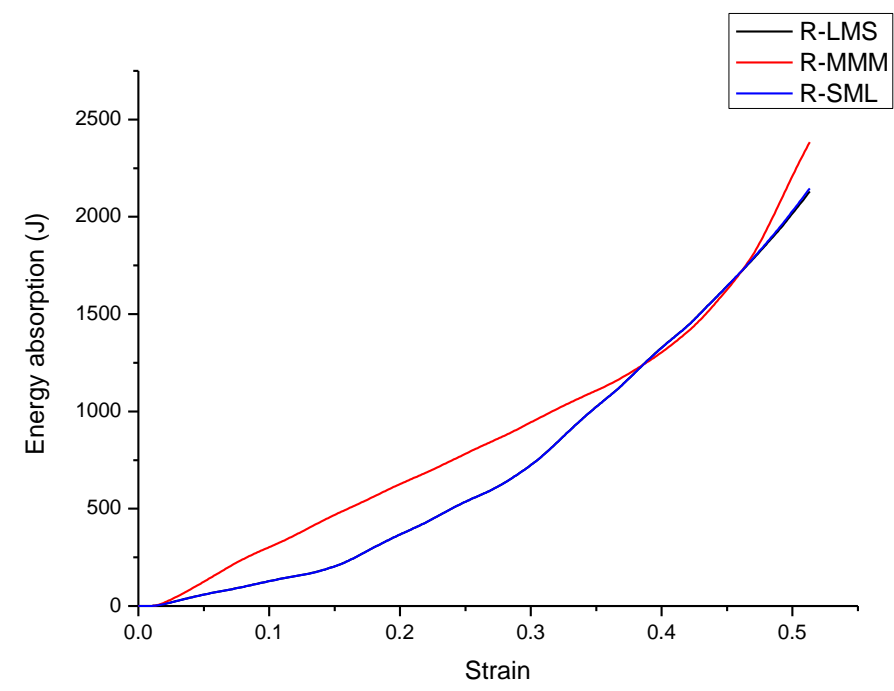

(a) $\mathrm{RHH}$

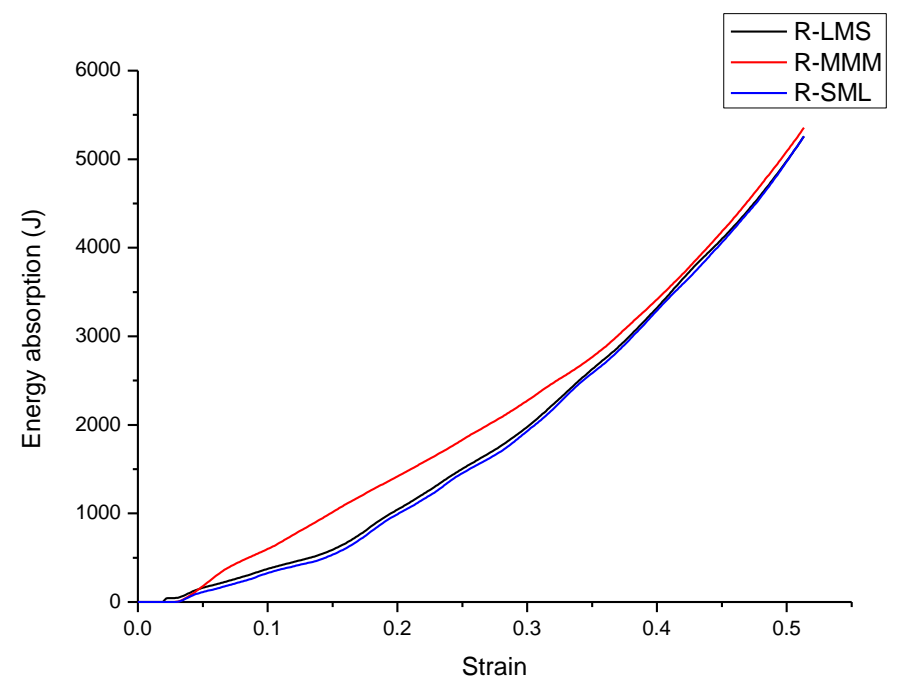

(b)RHT

Fig. 18. Comparsion of energy absorption of different gradient configurations of hierarchical sandwich panels under impact velocity of 1m/s:(a) RHH; (b)RHT. 


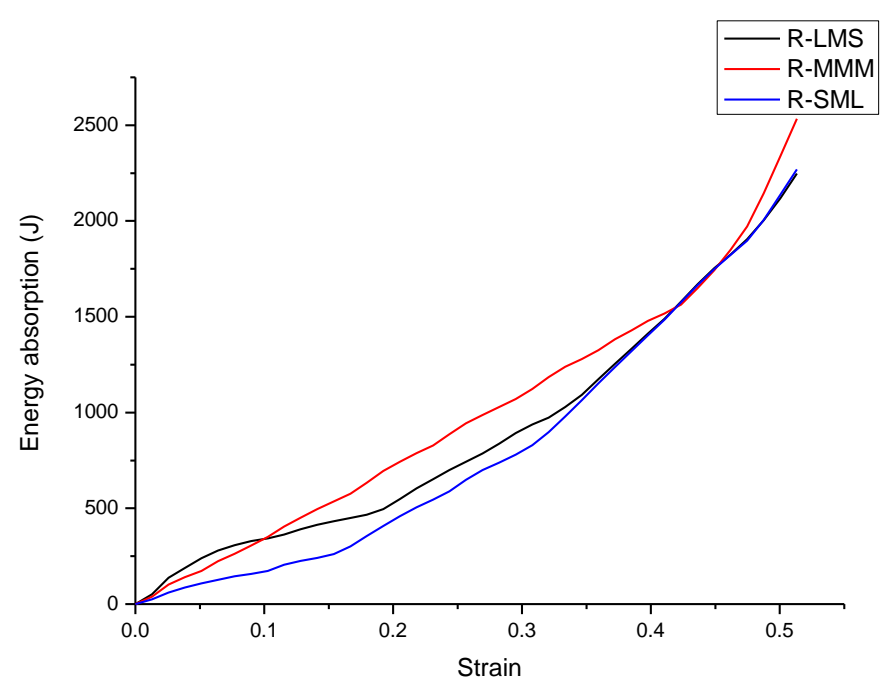

(a) RHH

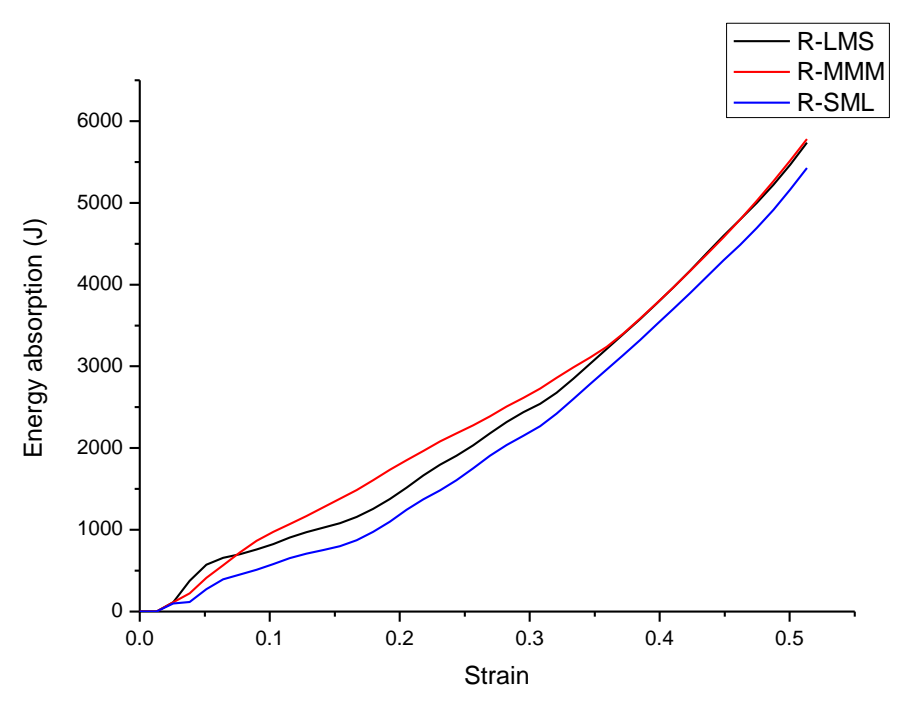

(b)RHT

Fig. 19. Comparsion of energy absorption of different gradient configurations of hierarchical sandwich panels under impact velocity of 10m/s:(a) RHH; (b)RHT.

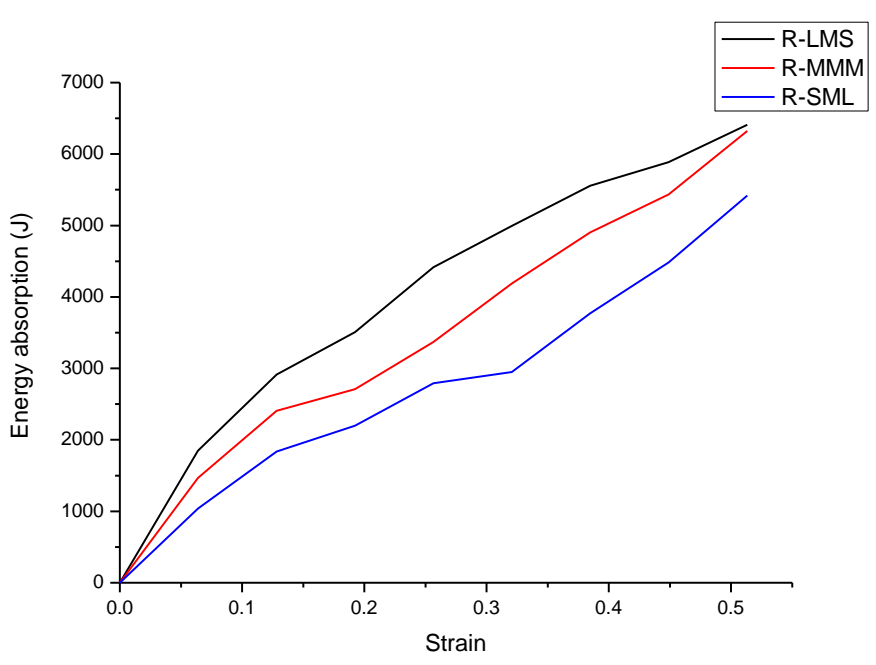

(a) RHH

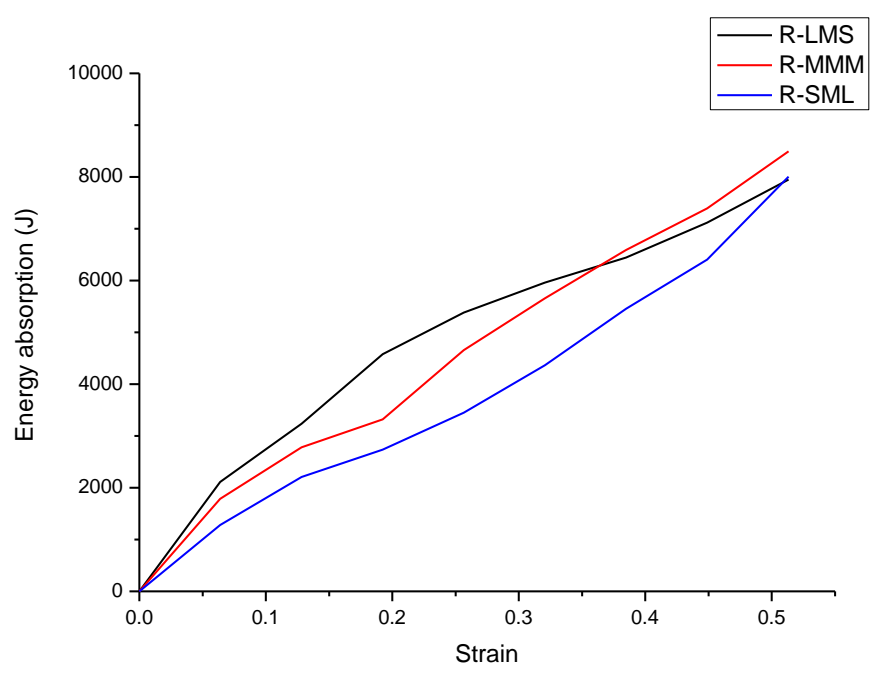

(b)RHT

Fig. 20. Comparsion of energy absorption of different gradient configurations of hierarchical sandwich panels under impact velocity of 50m/s:(a) RHH; (b)RHT.

The mechanisms of energy absorption are explained as follows. For quasi-static conditions, the inertia effect can be ignored, which result in that the deformation always starts from the thinnest cores and then spread to the thicker cores as shown in Fig. 14-15. This situation explains that the graded configurations of R-LMS and 
R-SML almost absorb the same energy. Additionally, the thicker cores dissipate more energy than the thinner cores in crushing process, which can explain that the ungraded panels absorb more energy than graded panels. Under low impact velocity, the collapse starts from the impact side owing to the inertia effect and then transmits from the thinnest cores to the thickness cores. As shown in Fig. 21, the upper core of the R-LMS is slightly deformed first, and then the thinnest core begins to collapse. On the other hand, both the R-MMM and R-SML start collapse from the upper core. This is the reason why the R-LMS absorbs the maximum energy in the early strain, and then it absorbs less energy compared with R-MMM. Interestingly, the upper core of R-SML has a larger deformation compared with the upper core of R-MMM with the same crushing distance, which can be explained that the thinnest core becomes more deformable due to the gradient. Under high impact loading, the deformation always starts from the impact side and propagates layer by layer subsequently because of the inertia effect as shown in Fig. 22. For configurations of LMS, there are more mass distributed in the impact side which leads to the highest energy absorption ability. Therefore, a rational arrangement of graded cores would improve the plastic energy dissipation ability, and enhance the impact resistance of structures.

(a)

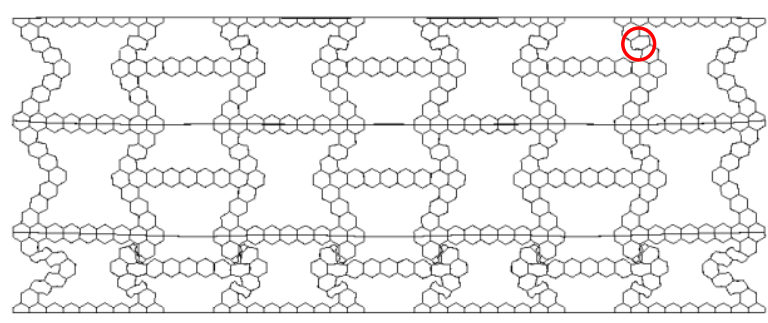


(b)

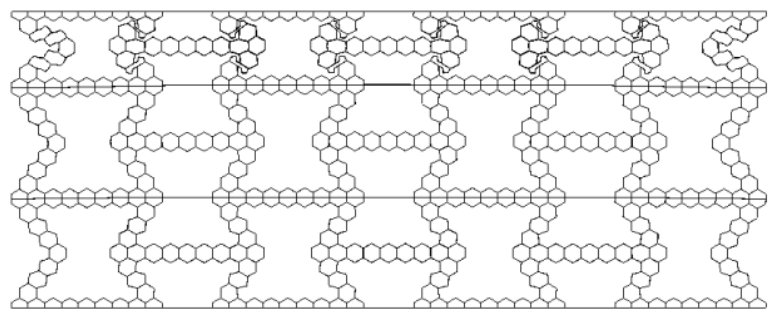

(c)

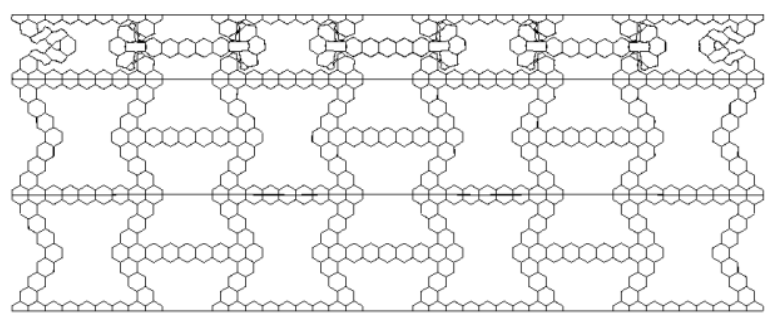

Fig. 21 Comparsion of deformation pattern of RHH hierarchical sandwich panels under impact velocity of 10m/s: (a) R-LMS; (b)R-MMM; (c) R-SML.

(a)

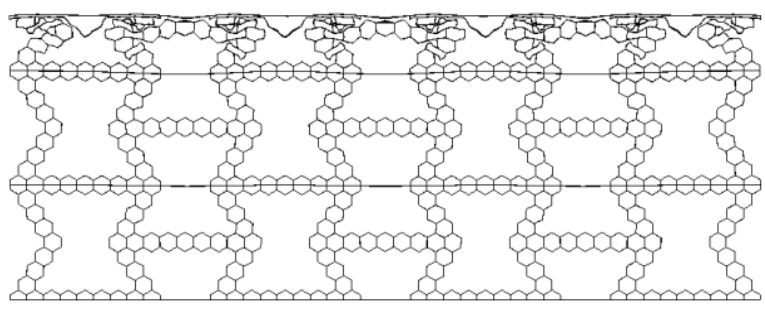

(b)

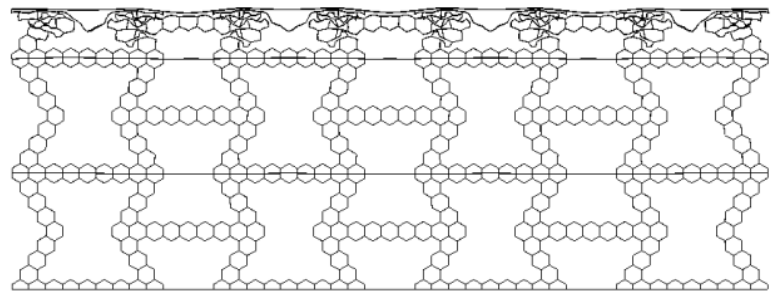

(c)

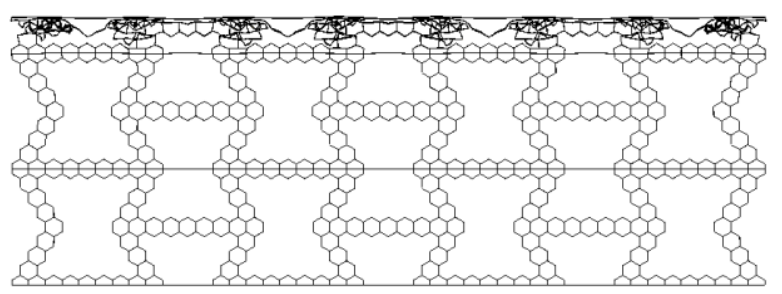

Fig. 22 Comparsion of deformation pattern of RHH hierarchical sandwich panels under impact velocity of 50m/s: (a) R-LMS; (b)R-MMM; (c) R-SML.

\subsection{Effect of the arranged orientations}

The arranged orientations of sandwich cores have a remarkable influence on crushing performance of the sandwich structures [35]. In this section, the influence of 
arranged orientations of hierarchical sandwich cores on the crushing response is investigated. There are two arranged orientations to present here: one is regularly-arranged orientation shown in Figs. 3-5(a), the other is cross-arranged orientation presented in Figs. 3-5(b).

\subsubsection{Effect of arranged orientations on crushing force}

As shown in Fig. 23, the comparison of crushing force between regularly-arranged orientation and cross-arranged orientation of RHT hierarchical sandwich panels with different configurations are presented. It is easy to see that the tendency of crushing force of the two arranged orientations is almost identical. It is clear that the graded hierarchical sandwich panels with cross-arranged orientation still have three stress plateaus corresponding to Figs. 16 and 17. Interestingly, the cross-arranged orientation of RHT sandwich panels makes the initial peak force earlier than regularly-arranged orientation, while the different arranged orientations of RHH sandwich cores basically do not affect the initial peak force, which can be seen from Fig. 24.

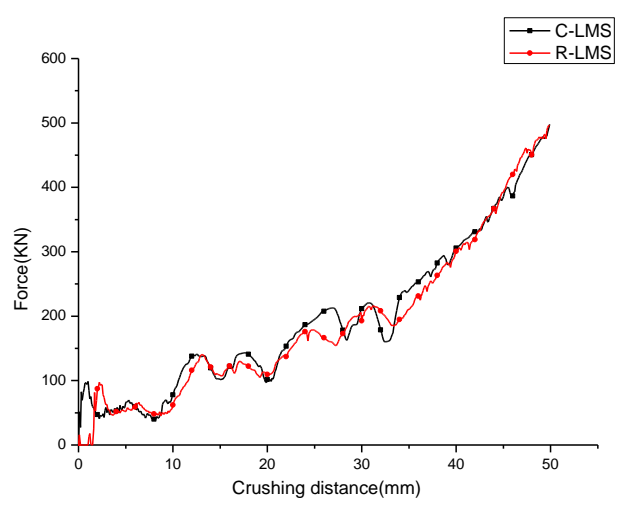

(a) Configuration of LMS

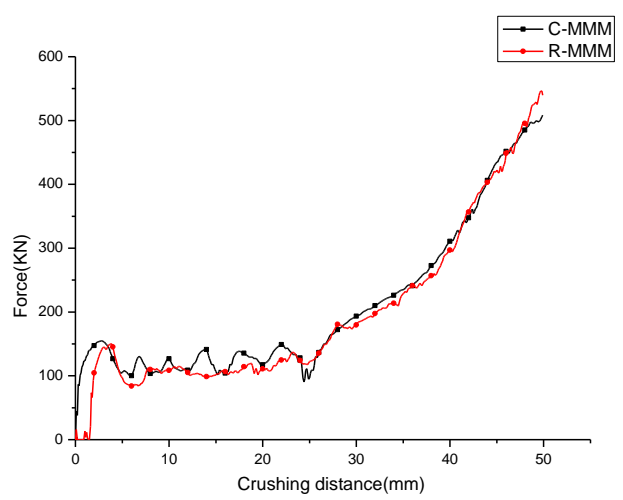

(b) Configuration of MMM

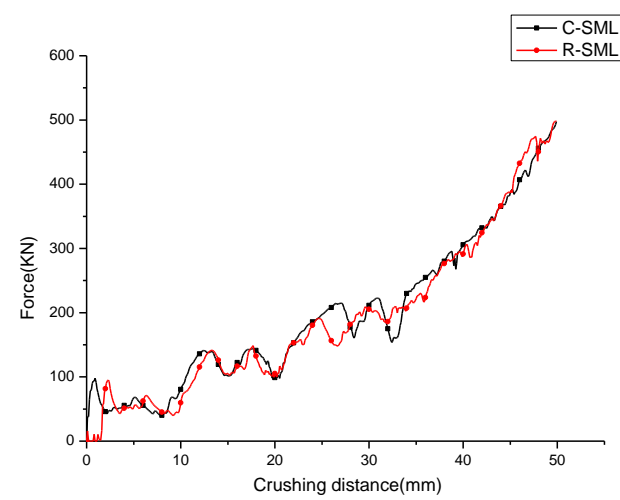

(c) Configuration of SML

Fig. 23. Comparison of crushing force of graded RHT sandwich panels with different arranged orientations. 


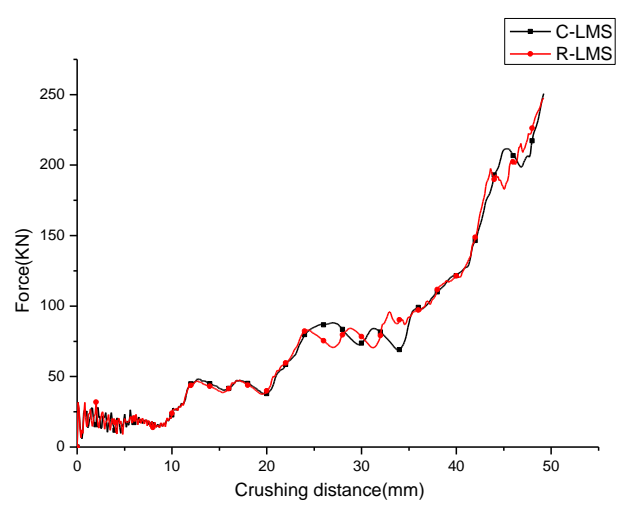

(a) Configuration of LMS

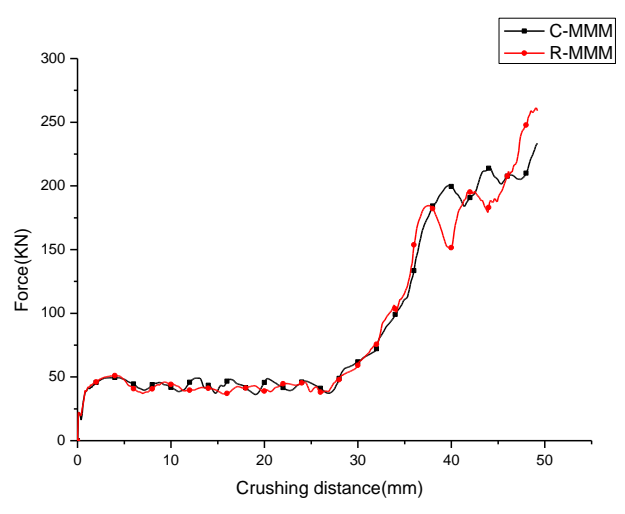

(b) Configuration of MMM

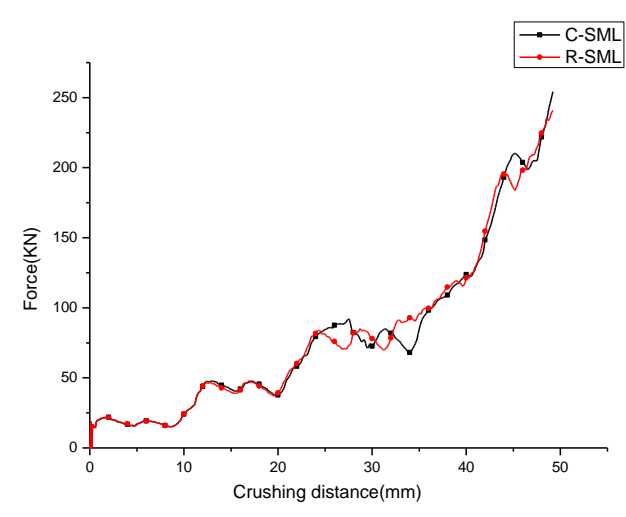

(c) Configuration of SML

Fig. 24. Comparison of crushing force of graded RHH sandwich panels with different arranged orientations.

\subsubsection{Effect of arranged orientations on energy absorption}

Fig. 25 shows the comparsion of MCF between regularly-arranged and cross-arranged orientations of different hierarchical sandwich panels at a crushing strain of $88.78 \%$. For the RHT hierarchical sandwich panels as shown in Fig. 25 (a), the cross-arranged orientation exhibits obviously higher value of MCF than regularly-arranged sandwich panels whatever the configuration is. However, the results of RHH hierarchical sandwich panels are different from the RHT. The MCF of cross-arranged orientation of RHH is slightly lower than regularly arranged orientation as shown in Fig. 25(b).

Similarly, Fig. 26 reveals that there exists consistency between the MCF and SEA of hierarchical sandwich panels. The SEA of cross-arranged orientation of RHT is higher than regularly-arranged orientation whereas the values of cross-arranged orientation of RHH are slightly lower than regularly-arranged orientation. It can be concluded that the arranged orientations have different effects on different 
hierarchical sandwich panel.

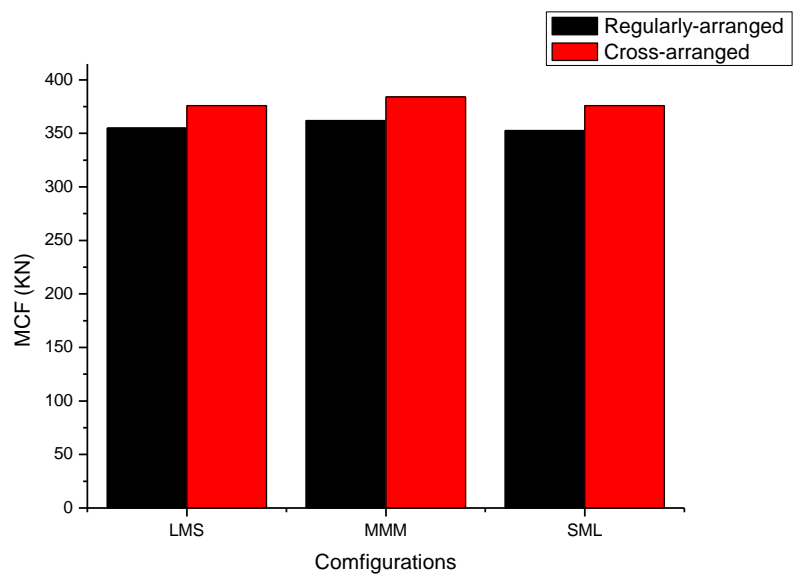

(a) RHT

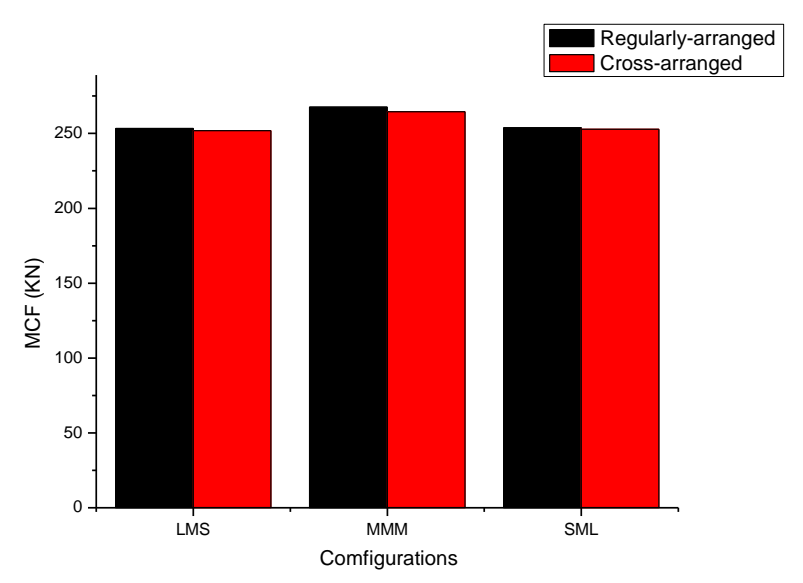

(b) $\mathrm{RHH}$

Fig. 25. Comparison of MCF of hierarchical sandwich panels with different arranged orientations.

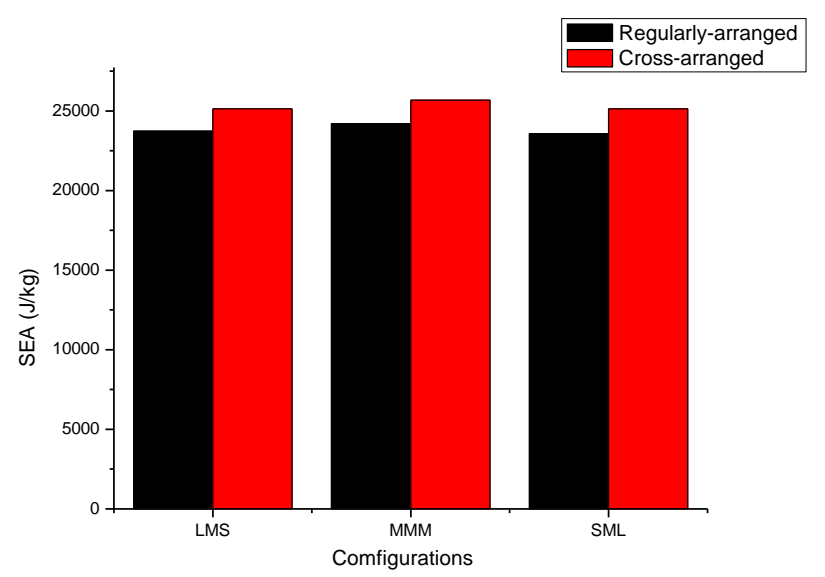

(a) RHT

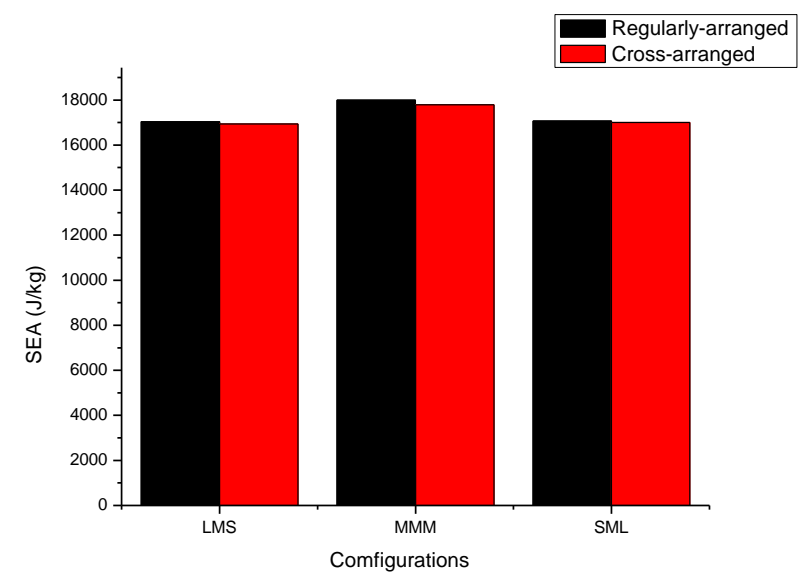

(b) RHH

Fig. 26. Comparison of SEA of hierarchical sandwich panels with different arranged orientations.

\subsection{Effect of the impact velocity}

The purpose of this section is to study the influences of different impact velocities on crushing properties of these hierarchical sandwich panels. The configuration of R-MMM is chosen as the type of specimens. Three different speeds $(1 \mathrm{~m} / \mathrm{s}, 10 \mathrm{~m} / \mathrm{s}, 50 \mathrm{~m} / \mathrm{s})$ are imposed on sandwich panels to investigate the crushing mechanism. 
As shown in Fig. 27, obvious differences in the deformation patterns can be seen with different impact velocities. Under quasi-static mode, the deformation occurs in the whole structure because of the neglect of the inertia effect. When increasing the impact velocity to $10 \mathrm{~m} / \mathrm{s}$, the densification mainly happens near the bottom of the specimen. When under high impact velocity of $50 \mathrm{~m} / \mathrm{s}$, the deformation can not propagate to the distal side without enough time, causing the deformation near the impact side.

(a)

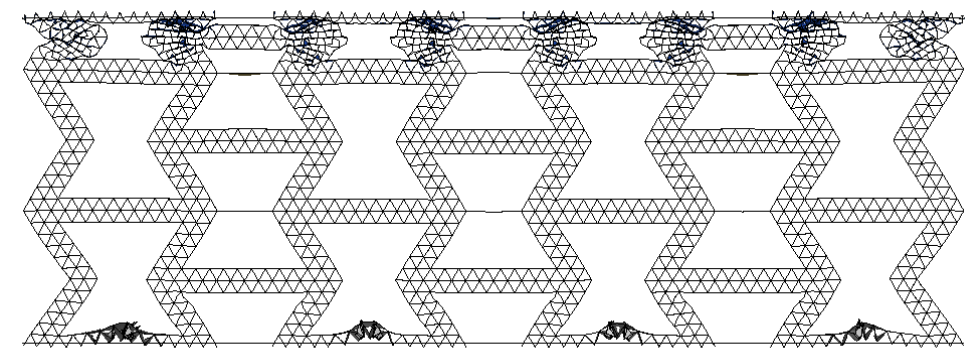

(b)

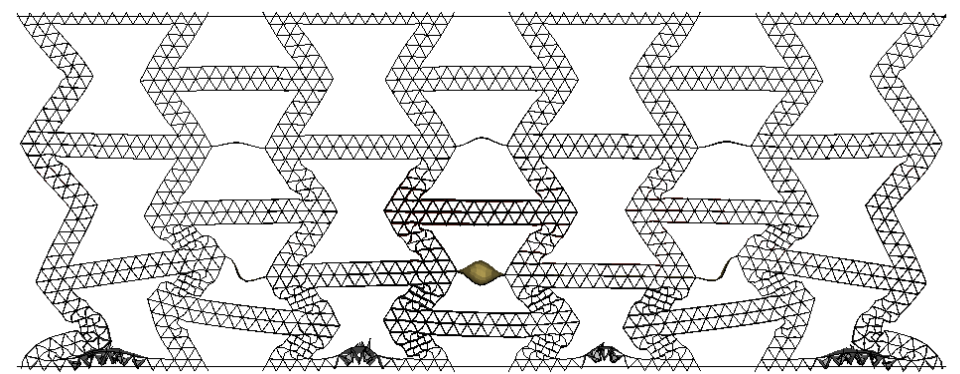

(c)

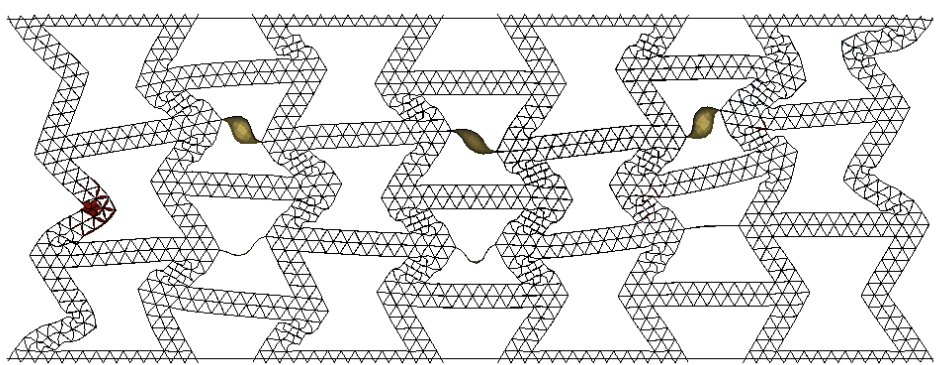

Fig. 27. Comparsion of deformation processes of RHT hierarchical sandwich panels under different impact velocities:(a) $50 \mathrm{~m} / \mathrm{s}$; (b) $10 \mathrm{~m} / \mathrm{s}$; (c) $1 \mathrm{~m} / \mathrm{s}$.

Since the MCF is the key indicators of energy absorption capacity, it can be used to compare the effect of gradient on crushing. MCF-strain curves are employed 
to evaluate the crushing properties of hierarchical sandwich panels. The impact velocity has a very important effect on the crushing performances of these sandwich panels. As shown in Fig. 28, the value of MCF of sandwich structures increases with the impact speed. It is apparent from this figure that the MCF values of RHT sandwich panels are always higher than RHH under the same impact velocity, which means the higher energy absorption capacity.

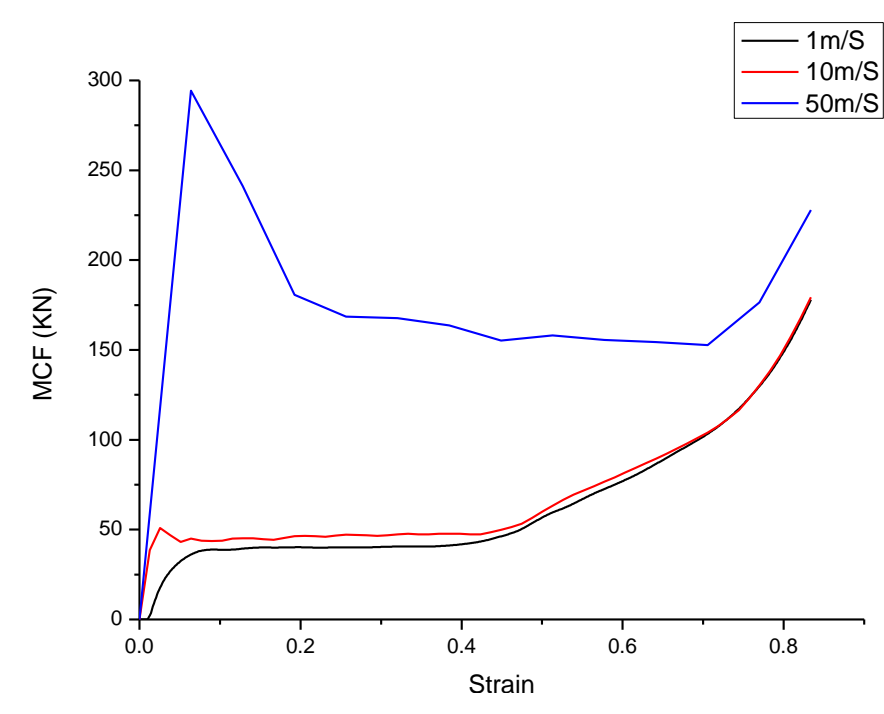

(a) RHH

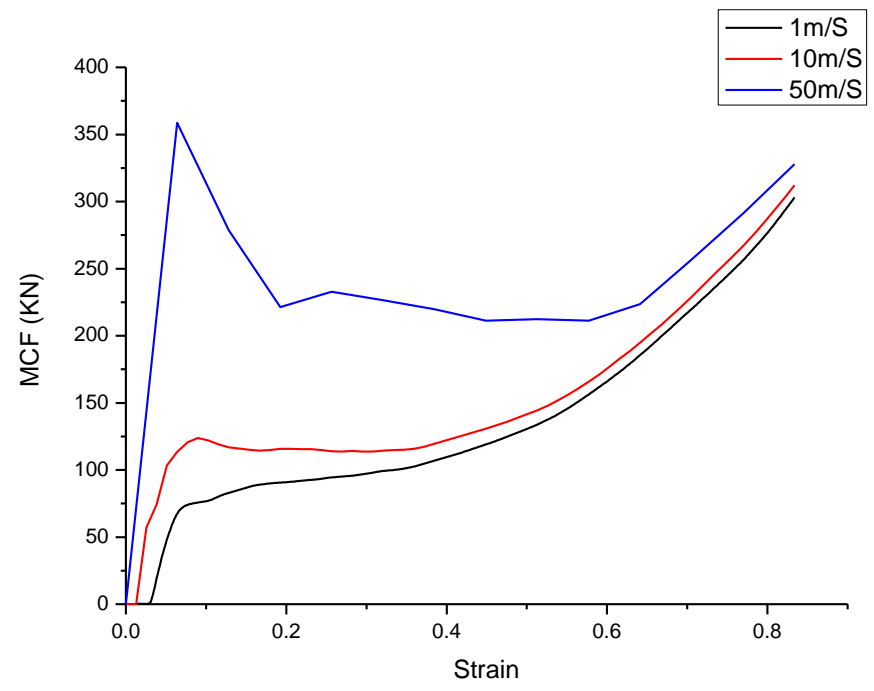

(b)RHT

Fig. 28. Comparsion of MCF of hierarchical sandwich panels under different impact velocities:(a) RHH; (b)RHT.

\section{Concluding remarks}

This paper proposed two novel re-entrant hierarchical sandwich panels, and their crashworthiness performance has been investigated in this work. A comparison between the re-entrant hierarchical sandwich panels and re-entrant sandwich panel has been conducted to study their energy absorption ability. Furthermore, the parametric analysis based on numerical analysis has been carried out to discuss the effects of the gradient, arranged orientation of hierarchical sandwich cores and the impact velocities. Based on the results among the sandwich panels, the following conclusions can be 
drawn:

(1) Analytical analysis indicates that for the given configurations of the honeycombs, the values of the relative in-plane stiffness of the RHH and RHT have been increased by 1.3 times and 6.7 times respectively compared with $\mathrm{RH}$ at a relative density of 0.1 . Therefore, the introduction of hierarchy can greatly improve the Young's modulus of a honeycomb.

(2) The introduction of hierarchy into sandwich panel can greatly improve the energy absorption capacity. The values of MCF of RHT and RHH sandwich panels are $298.2 \%$ and $208.2 \%$ higher than that of $\mathrm{RH}$ respectively, and the RHT sandwich panel improves SEA by $269.1 \%$, and RHH sandwich panel improves SEA by $203.1 \%$ above RH sandwich panel at a displacement of $70 \mathrm{~mm}$ with the configuration of R-MMM.

(3) The graded hierarchical sandwich panels have three crushing stress plateaus while the ungraded hierarchical sandwich panels only have one crushing stress plateau, and the order of the graded hierarchical cores would not affect the tendency of crushing stress.

(4) The graded hierarchical sandwich panels can significantly reduce the initial peak force. The configuration of LMS and SML of RHT sandwich panels can reduce the initial peak force by $35.1 \%$ and $36.6 \%$ respectively, and the R-SML and R-LMS configuration of RHH can reduce initial peak force by $56.5 \%$ and $38.0 \%$ respectively.

(5) Results reveal that the graded hierarchical sandwich panels with 
configuration of LMS have a better impact resistance than other sandwich panels when subjected to higher impact velocity, while the ungraded sandwich panels perform better under low impact velocity.

(6) The RHT hierarchical sandwich panels with cross-arranged orientation can improve the impact performance.

\section{Acknowledgments}

The project is supported by the Foundation for Innovative Research Groups of the National Natural Science Foundation of China (Grant No. 51621004) and the Natural Science Foundation of China (Grant No. U1864207), the Opening Project of the Guangxi Key Laboratory of Automobile Components and Vehicle Technology of Guangxi University of Science and Technology (No. 2017GKLACVTKF01).

\section{References}

[1] X. Yang, J. Ma, Y. Shi, Y. Sun, J. Yang, Crashworthiness investigation of the bio-inspired bi-directionally corrugated core sandwich panel under quasi-static crushing load, Materials \& Design, 135 (2017) 275-290.

[2] S. Hou, S. Zhao, L. Ren, X. Han, Q. Li, Crashworthiness optimization of corrugated sandwich panels, Materials \& Design, 51 (2013) 1071-1084.

[3] B.G. Vijayasimha Reddy, K.V. Sharma, T. Yella Reddy, Deformation and impact energy absorption of cellular sandwich panels, Materials \& Design, 61 (2014) 217-227.

[4] D. Zhang, Q. Fei, P. Zhang, Drop-weight impact behavior of honeycomb sandwich panels under a spherical impactor, Composite Structures, 168 (2017) 633-645.

[5] Q. Qin, X. Zheng, J. Zhang, C. Yuan, T.J. Wang, Dynamic response of square sandwich plates with a metal foam core subjected to low-velocity impact, International Journal of Impact Engineering, 111 (2018) 222-235.

[6] T. Liu, S. Hou, X. Nguyen, X. Han, Energy absorption characteristics of sandwich structures with composite sheets and bio coconut core, Composites Part B: Engineering, 114 (2017) 328-338.

[7] Y. Hu, W. Li, X. An, H. Fan, Fabrication and mechanical behaviors of corrugated lattice truss composite sandwich panels, Composites Science and Technology, 125 (2016) 114-122.

[8] W. Li, F. Sun, W. Wei, D. Liu, X. Zhang, M. Li, H. Fan, Fabrication and testing of composite corrugated-core sandwich cylinder, Composites Science and Technology, 156 (2018) 127-135.

[9] A.A. Nia, S. Mokari, M. Zakizadeh, M. Kazemi, Experimental and numerical investigations of the effect of cellular wired core on the ballistic resistance of sandwich structures, Aerospace Science and 
Technology, 70 (2017) 445-452.

[10] M. Zarei Mahmoudabadi, M. Sadighi, Experimental investigation on the energy absorption characteristics of honeycomb sandwich panels under quasi-static punch loading, Aerospace Science and Technology, (2019).

[11] W. Zhang, S. Yin, T.X. Yu, J. Xu, Crushing resistance and energy absorption of pomelo peel inspired hierarchical honeycomb, International Journal of Impact Engineering, 125 (2019) 163-172.

[12] M.N. Velea, S. Lache, Energy absorption of all-PET 2nd order hierarchical sandwich structures under quasi-static loading conditions, Thin-Walled Structures, 138 (2019) 117-123.

[13] X. An, H. Fan, Hybrid design and energy absorption of luffa-sponge-like hierarchical cellular structures, Materials \& Design, 106 (2016) 247-257.

[14] H.H. Tsang, S. Raza, Impact energy absorption of bio-inspired tubular sections with structural hierarchy, Composite Structures, 195 (2018) 199-210.

[15] F. Sun, C. Lai, H. Fan, In-plane compression behavior and energy absorption of hierarchical triangular lattice structures, Materials \& Design, 100 (2016) 280-290.

[16] Y. Zhang, X. Xu, J. Wang, T. Chen, C.H. Wang, Crushing analysis for novel bio-inspired hierarchical circular structures subjected to axial load, International Journal of Mechanical Sciences, 140 (2018) 407-431.

[17] W. Li, Y. Luo, M. Li, F. Sun, H. Fan, A more weight-efficient hierarchical hexagonal multi-cell tubular absorber, International Journal of Mechanical Sciences, 140 (2018) 241-249.

[18] H.L. Tan, Z.C. He, K.X. Li, E. Li, A.G. Cheng, B. Xu, In-plane crashworthiness of re-entrant hierarchical honeycombs with negative Poisson's ratio, Composite Structures, 229 (2019) 111415.

[19] X. Jin, Z. Wang, J. Ning, G. Xiao, E. Liu, X. Shu, Dynamic response of sandwich structures with graded auxetic honeycomb cores under blast loading, Composites Part B Engineering, 106 (2016) 206-217.

[20] Z.-X. Lu, X. Li, Z.-Y. Yang, F. Xie, Novel structure with negative Poisson's ratio and enhanced Young's modulus, Composite Structures, 138 (2016) 243-252.

[21] C. Qi, A. Remennikov, L.Z. Pei, S. Yang, Z.H. Yu, T.D. Ngo, Impact and close-in blast response of auxetic honeycomb-cored sandwich panels: experimental tests and numerical simulations, Composite Structures, 180 (2017) 161-178.

[22] W. Liu, N. Wang, L. Tao, Z. Lin, In-plane dynamic crushing of re-entrant auxetic cellular structure, Materials \& Design, 100 (2016) 84-91.

[23] M. Fu, Y. Chen, L. Hu, A novel auxetic honeycomb with enhanced in-plane stiffness and buckling strength, Composite Structures, 160 (2017) 574-585.

[24] N.D. Duc, K. Seung-Eock, N.D. Tuan, P. Tran, N.D. Khoa, New approach to study nonlinear dynamic response and vibration of sandwich composite cylindrical panels with auxetic honeycomb core layer, Aerospace Science and Technology, 70 (2017) 396-404.

[25] X. Lan, S. Feng, Q. Huang, T. Zhou, A comparative study of blast resistance of cylindrical sandwich panels with aluminum foam and auxetic honeycomb cores, Aerospace Science and Technology, (2019).

[26] Y. Guo, J. Zhang, L. Chen, B. Du, H. Liu, L. Chen, W. Li, Y. Liu, Deformation behaviors and energy absorption of auxetic lattice cylindrical structures under axial crushing load, Aerospace Science and Technology, 98 (2020) 105662.

[27] Q. Gao, W.-H. Liao, L. Wang, On the low-velocity impact responses of auxetic double arrowed honeycomb, Aerospace Science and Technology, 98 (2020) 105698. 
[28] C. Li, H.-S. Shen, H. Wang, Thermal post-buckling of sandwich beams with functionally graded negative Poisson's ratio honeycomb core, International Journal of Mechanical Sciences, 152 (2019) 289-297.

[29] C.M. Taylor, C.W. Smith, W. Miller, K.E. Evans, Functional grading in hierarchical honeycombs: Density specific elastic performance, Composite Structures, 94 (2012) 2296-2305.

[30] C.M. Taylor, C.W. Smith, W. Miller, K.E. Evans, The effects of hierarchy on the in-plane elastic properties of honeycombs, International Journal of Solids and Structures, 48 (2011) 1330-1339.

[31] S.C. Pradhan, T. Murmu, Thermo-mechanical vibration of FGM sandwich beam under variable elastic foundations using differential quadrature method, Journal of Sound and Vibration, 321 (2009) 342-362.

[32] D. Xiao, Z. Dong, Y. Li, W. Wu, D. Fang, Compression behavior of the graded metallic auxetic reentrant honeycomb: Experiment and finite element analysis, Materials Science and Engineering: A, 758 (2019) 163-171.

[33] G. Sun, G. Li, S. Hou, S. Zhou, W. Li, Q. Li, Crashworthiness design for functionally graded foam-filled thin-walled structures, Materials Science and Engineering: A, 527 (2010) 1911-1919.

[34] G. Wang, L.Y. Kou, X.U. Cong-Chang, Y.E. Tuo, L.I. Luo-Xing, Finite Element Modeling for Compression Fracture Behavior of6000Aluminum Alloys Thin-Walled Structure, Materials for Mechanical Engineering, (2016).

[35] S. Hou, C. Shu, S. Zhao, T. Liu, X. Han, Q. Li, Experimental and numerical studies on multi-layered corrugated sandwich panels under crushing loading, Composite Structures, 126 (2015) 371-385.

[36] L.J. Gibson, M.F. Ashby, Cellular solids: structure and properties, Cambridge university press, 1999.

[37] Y. Sun, N.M. Pugno, In plane stiffness of multifunctional hierarchical honeycombs with negative Poisson's ratio sub-structures, Composite Structures, 106 (2013) 681-689.

[38] Y. Sun, B. Wang, N. Pugno, B. Wang, Q. Ding, In-plane stiffness of the anisotropic multifunctional hierarchical honeycombs, Composite Structures, 131 (2015) 616-624. 